\title{
VIBRATIONAL CONTROL OF CHEMICAL REACTORS: SELECTIVITY ENHANCEMENT, STABILIZATION AND IMPROVEMENT OF TRANSIENT BEHAVIOR
}

\author{
Final Report \\ for the period of April 1, 1987 - June 30, 1990
}

Ali Cinar

Department of Chemical Engineering Illinois Institute of Technology

Chicago, IL 60616

September 1990

Prepared for

THE U. S. DEPARTMENT OF ENERGY

AGREEMENT NO. DE-FG02-87ER13696

MnOTER

DISTRIBUTIONN OF THIS DOCUMENT IO UN: 


\section{NOTICE}

This report was prepared as an account of work sponsored by the United States Government. Neither the United States nor the Department of Energy, nor any of their employees, nor any of their contractors, subcontractors or their employees, makes any warranty, express or implied, or assumes any legal liability or respon- sibility for the accuracy, completeness, or usef-iness of any information, apparatus, product or process disclosed or represents that its use would not infringe privately-owned rights.

\section{DISCLAIMER}

\footnotetext{
This report was prepared as an account of work sponsored by an agency of the United States Government. Neither the United States Government nor any agency thereof, nor any of their employees, $r$ akes any warranty, express or implied, or assumes any legal liability or responsibility for the accuracy, compleceness, or usefulness of any information, apparatus, product, or process disclosed, or represents that its use would not infsinge privately owned rights. Reference herein to any specific commercial product, process. or service by trade name, trademark, manufacturer, or otherwise does not necessarily constitute or imply its endorsement, recommendation, or favoring by the United States Government or any agency thereof. The views and opinions of authors expressed herein do not necessarily state or reflect those of the United States Government or any agency thereof.
} 


\section{TABLE OF CONTENTS}

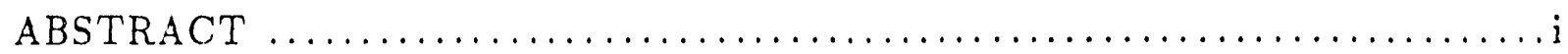

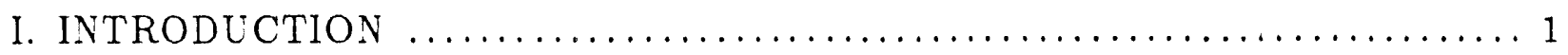

II. SELECTIVITY AND YIELD IMPROVEMENT BY

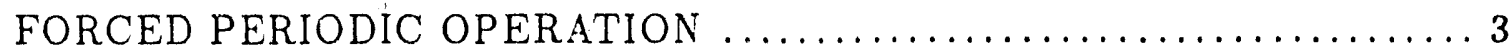

Assessment of Forced Periodic Operation by Vibrational Control Method ... 3

Assersment of Forced Periodic Operation by Shooting Algorithm Approach . . 6

III. CLASSI: ICATION STEADY STATE REACTOR BEHAVIOR BY

NUMIERICAL SINGULARITY AND BIFLRCATION ANALYSIS $\ldots \ldots \ldots .9$

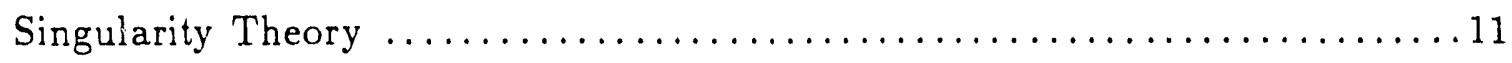

Recognition of Codimension 1 Singularity

Continuation of Codimension 1 Singularity

Computational Details ..................................... 15

Bifurcation Behavior of Feedback Controlled Reactors ................. 15

Bifurcation Behavior of Reactors Under Forced Periodic Operation ..........18

IV. VIBRATIONAL-FEEDBACK CONTROL OF CHEMICAL REACTORS $\ldots \ldots .19$

'ibrational-Feedback Control Method ...........................20

The Vibrational-Feedback Controller

The A veraged Closed Loop System

Tuning the Vibrational-Feedback Controller

Vitrational Feedback Control of a Tubular Reactor $\ldots \ldots \ldots \ldots \ldots \ldots \ldots . \ldots 23$

The Reactor Model

Dynamic Response of the Reactor

Conclusions

V. CONCLUSIONS AND FUTURE RESEARCH DIRECTIONS $\ldots \ldots \ldots \ldots \ldots \ldots 26$

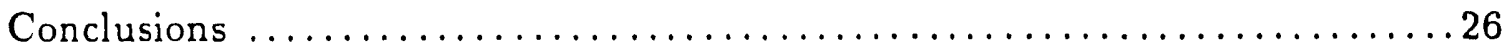

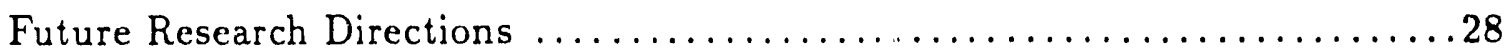

VI. LIST OF PUBLICATIONS AND PRESENTATIONS RESULTING FROM

THE PROJECT AND EDUCATIONAL ACTIVITIES $\ldots \ldots \ldots \ldots \ldots \ldots . \ldots 29$

VII. REFERENCES

APPENDICES

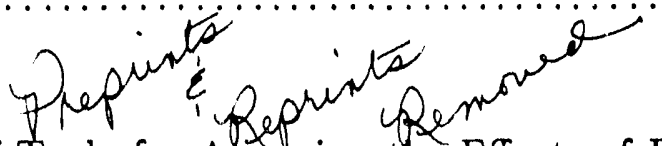

1. A Comparative Study of Tools for Assessing the Effects of Forced Periodic Catalytic Reactor Operation

2. A Numerical Method for Determining Optimal Parameter Values In Forced Periodic Operation

3. Numerical Singularity Analysis

4. Numerical Singularity Analysis of Feedback Controlled Chemical Reactors

5. Dynamic Behavior of Periodic Feedback Control Systems 


\begin{abstract}
The effects of vibrational control on selectivity and yield of complex chemical reactions and on tubular packed-bed reactor lenavior have been studied.

i. Selectivity and yield improvement by forced periodic operation of complex chemical systems have been assessed by vibrational control approach and by a numerical approach. Vibrational control approach has provided good insight. The numerical approach is based on the computation of stable limit cycles by using a shooting algorithm. Since the reactor model is incorporated as a subroutine to the computer code developed, effects of forced periodic operation on various types of reactions and reactors can be assessed with ease. The program has been integrated in an optimization package for the selection of optimal operating conditions for maximizing the reactor yield.

The forced periodic operation of ethylene oxidation reaction system, a hydrocarbon partial oxidation reaction of commercial importance, has been studied using both techniques. Yield improvement of 23 percent tas been attained by periodic forcing of feed flow rate and ethylene feed concentration.

ii. A numerical singularity analysis technique has been developed and applied to openloop and feedback controlled tubular packed-bed reactors. The technique is applicable to various kinds of reactor systems and it can be used to classify their qualitatively different static bifurcation behaviors as a function of input variables such as feed temperature and concentration. Such a classification displays the existence of other attractors in the vicinity of the steady state around which a reactor would be subjected to forced periodic operation. Preliminary studies have been conducted to extend the technique to analyze and classify the bifurcation behavior of chemical reactors inder vibrational control.

iii. Low order vibrational-feedback controllers have been designed and a new tuning method has been used in vibrational-feedback control of chemical reactors. Vibrationalfeedback controllers allow zero relocation and consequently have th: potential of eliminating inverse response behavior due to right half-plane zeros. A vibrational-ieedback controller based on a reduced-order model of the $\mathrm{CO}$ oxidation reactor has greatly improved the gain margin and improved the averaged dynamic behavior. However, the inverse response behavior persisted in dynamic simulations of the actual system and excessive moves were expected of the manipulated variable.
\end{abstract}




\section{INTRODUCTION}

In this report, the results of a study on vibrational control of chemical reactors are reported and future research topics are identified. This work was sponsored by the Division of Engineering, Mathematical and Geosciences, Office of Basic Energy Sciences of the U.S. Department of Energ; and it was performed at the Department of Chemical Engineering of Illinois Institute of Technology.

Many chemical reactions of industrial importance such as ammonia synthesis, methanol synthesis, partial oxidation of hydrocarbons are car ried out in packed-bed tubular reactors. Often the behavior of these reactors is forecast based on limited amount of simulation studies and past operational experience. The lack of detailed knowledge about reactor behavior induces conservative operation of such reactors, resulting in loss of productivity and excessive use of energy. The rewards in operating those reactors under more efiective $o_{r}^{r}$ erating conditions are substantial. Yet without detailed investigation of what may happen under the new operating conditions, the modification of the operating conditions to previously unknown regions would be premature and ill-advised. First, the stability of the reactor operation under the new conditions must be assured. Next, the behavior of the reactor must be assessed when the operating conditions drift away from the new nominal operating conditions. These reactors are distributed parameter nonlinear systems which have multiple steady states under some range of operating conditions and sometimes a one degree change in the temperature of the reactor feed stream or reactor wall can cause reaction extinction, ignition or runaway. Hence, the investigation and classification of all possible steady states in the range of operating conditions are necessary. Finally, after assuring the stability of reactor operation and the lack of dangerous states in the vicinity of the operating point, the contribution of periodic forcing policies in improving the reactor productivity and reducing energy waste can be assessed.

There is a misconception among some process engineers in industry that industrial reactors do not have multiple steady state regions and unstable steady state regions. Not 
all reactors have such regions of operation. But if they exhibit pathological behavior in some range of operating conditions, this behavior can be identified either by thorough theoretical studies or by deliberate attempts to operate under conditions corresponding to pathological behavior. If this region of operating conditions is entered infrequently and accidentally during regular reactor operation, the behavior may not be identified properly and may be dismissed as an aberration. Yet such "aberrations" may include very dangerous as well as very lucrative operating points.

Our research on vibrational control of chemical reactors had to address the classification of steady state behavior problem in order to diagnose existence of pathological behavior. A vast literature exists for checking the stability of reactor operation. Yet, tools for computing all possible steady states under a set of operating conditions and classifying the different regions of operating conditions which yield qualitatively different reactor behaviors were scarce and inappropriate for tubular packed bed reactors. Consequently, part of our effort during the project period was focused on the development of tools to assess and classify reactor behavior. The numerical singularity analysis technique and bifurcation analysis tools [1] are the outcome of this activity. Stability analysis is included in these tools.

Vibrational control studies have focused on the contributions of periodic forcing of reactor inputs in yield and selectivity improvement of complex chemical reactions and on stability and dynamic behavior improvement in packed-bed tubular reactors. The selectivity and yield assessment studies considered single as well as multiple input oscillations. Since the extension of the averaging method became difficult as the complexity of the reaction mechanism and/or the reactor configuration increased, a different analysis tool based on the romputation of stable limit cycles by shooting algorithms [2] was developed.

The study of vibrational feedback-controllers in improving dynamic reactor behavior necessitated the development of efficient controller design methods. A control system design procedure based on root-locus analysis was developed [3] and utilized in this study. 
Vibrational-feedback controllers can relocate the closed-loop zeros of process. Since right half-plane zeros are the cause of inverse response behavior, the possibility of eliminating inverse response behavior in packed-bed tubular reactors by vibrational-feedback controllers was investigated.

The report is structured as follows. The techniques developer and the results obtained in selectivity and yield enhancement by forced periodic operation are discussed in Chapter II. The following two chapters are devoted to numerical singularity analysis techniques and its application to chemical reactors (Chapter III), and to vibrational-feedback control of chemical reactors (Chapter IV), respectively. Next, the conclusions and future research directions identified are summarized in Chapter $\mathrm{V}$. The list of publications and presentations resulting from the project are stated in Chapter VI, followed by the listing of references in Chapter VII. The major unpublished maruscripts resulting from this project are included in the Appendix. Since the details of various novel techniques and resuits are given in these publications, the discussion in the report is focused on the key issues. Appropriate references to these publications are provided for the audience ir erested in the details of the various contributions.

\section{SELEC'TIVITY AND YIELD IMPROVEMENT BY FORCED PERIODIC OPERATION}

\section{Assessment of Effects of Periodic Forcing by Vibrational Control Method}

Forced periodic operation of chemical reactors for conversion improvement and selec. tivity enhancement has been studied for about three decades. Most studies focused on the selection of operating conditions that maximize the improvements through experimental studies. Although they provided results which indicated spectacular improvements and convinced the community about the potential merits of research in this area, the extrapolation of their results to industrial systems is difficult. Furthermore, the selection of the range of operating conditions which enhance conversion and/or selectivity is rather time 
consuming and the results can not be extended to other systems. An alternative is to carry out theoretical itudies for finding the range of operating conditions which show promise and then validate them experimentally.

The vibrational control technique provides a theoretical analysis tool based on the method of averaging $[4,5]$. It does not suffer from the restrictive assumptions such as small amplitude perturbation assumption of the П-criterion approach. In a previous DOE Grant [6], it has been applied to increase the stabilized operation region of a CSTR [7] and has been useful in assessing the effects of multiple input forcings $[8,9]$. The vibrational control method generates averaged system equations for a system subject to periodic forcing around a stable equilibrium point. Consequently, the task of solving the set of equations representing a dynamical system with parametric oscillations is reduced to the task of solving the averaged system equations. The solution of the averaged system equations at a specific set of operating̈ conditions yields the average values of the system variables, providing considerable savings in computational effort over computing the stable limit cycle at the same operating conditions and taking the average values of the system variables over one period. The method of anaiysis is summarized in the Appendix of the manuscript in Appendix 1.

In this study, the vibrational control approach is used to investigate the effects of single and multiple input periodic forcings on a packed-bed internal recycle reactor where ethylene is converted to ethylene oxide. Partial oxidation of ethylene has commercial applications and is representative of the series-parallel partial oxidation reactions of hydrocarbons. Such reactions have important industrial applications such as phthallic anhydride production from o-xylene, maleic anhydride production from butene and acrolein prcduction from propylene [10]. In these reactions, the partial oxidation reaction competes with a parallel toial oxidation reaction which yields carbon dioxide and water $[2,10]$. There is also a consecutive reaction causing the product of the partial oxidation reaction to dirintegrate into carbon dioxide and water. These two reactions are an order of magnitude more 


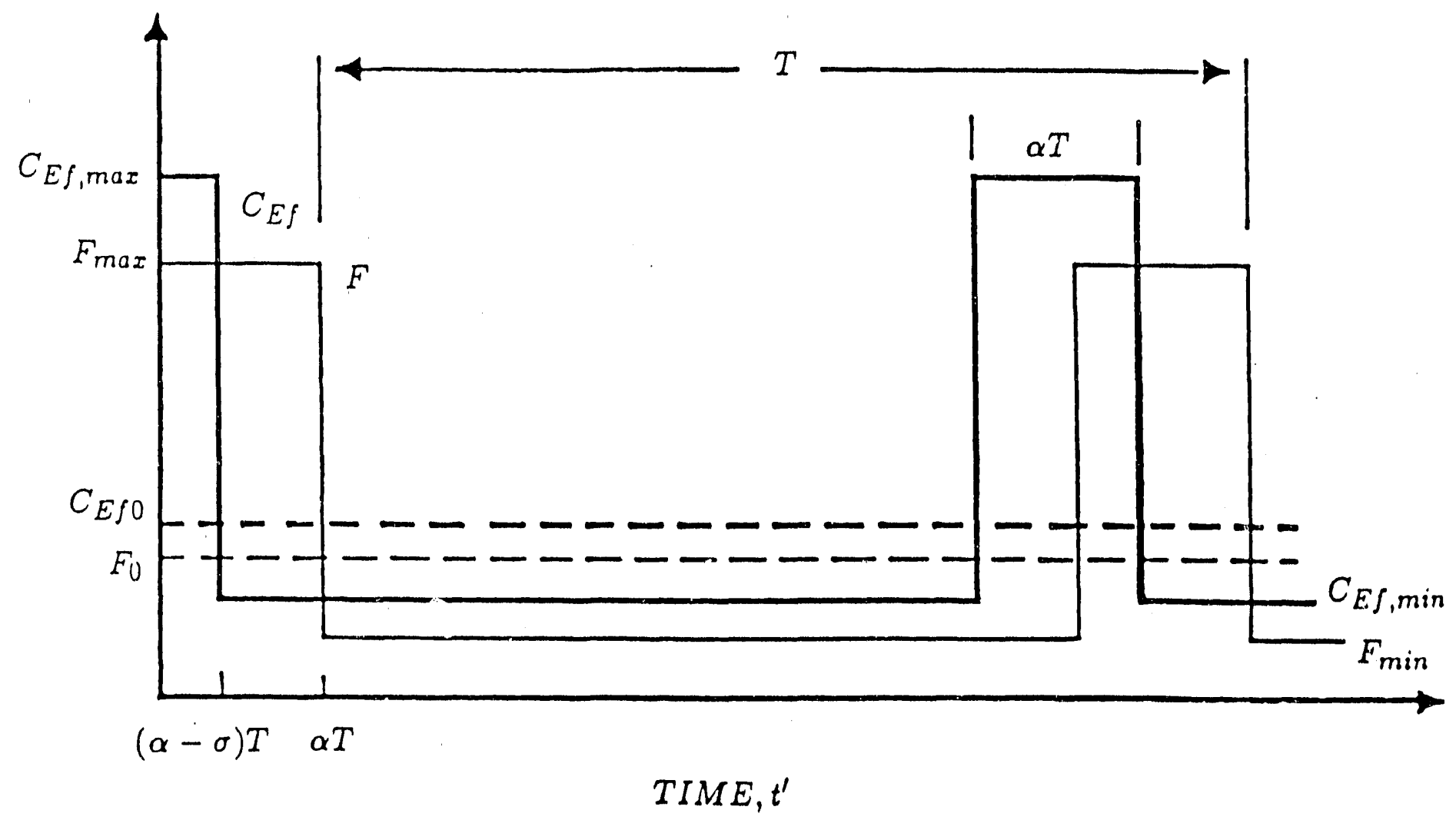

Figure 1. The pattern of nonsymmetric rectangular forcing function. 
Table 1. Nonisothermal CSTR operation. Comparison of vibrational control and shooting algorithm results.

$$
\begin{array}{cc}
\text { Adiabatic CSTR } & \text { Nonadiabatic CSTR } \\
\left(F_{0}=4000 \mathrm{sccm}, C_{E f 0}=0.35 \%\right) & \left(F_{0}=3500 \mathrm{sccm}, C_{E f 0}=1.0 \%\right)
\end{array}
$$

\begin{tabular}{lclcl}
\hline Parameters & $\begin{array}{l}\text { Vibrational } \\
\text { Control }\end{array}$ & $\begin{array}{l}\text { Shooting } \\
\text { Algorithm }\end{array}$ & $\begin{array}{l}\text { Vibrational } \\
\text { Control }\end{array}$ & $\begin{array}{l}\text { Shooting } \\
\text { Algorithm }\end{array}$ \\
\hline steady & $\bar{C}=0.220$ & 0.221 & $\bar{C}=0.097$ & 0.098 \\
state & $\bar{Y}=8.14 \%$ & $8.15 \%$ & $\bar{Y}=3.29 \%$ & $3.32 \%$ \\
$T=30, \alpha=0.2$ & 0.226 & 0.229 & 0.097 & 0.098 \\
$R_{f}=5$ & 8.41 & 8.44 & 3.29 & 3.31 \\
$T=30, \alpha=0.2$ & 0.200 & 0.203 & 0.091 & 0.093 \\
$R_{f}=5, R_{c}=5$ & 7.59 & 7.62 & 3.19 & 3.17 \\
$\sigma=0.0$ & & & & \\
$T=30, \alpha=0.2$ & 0.241 & 0.246 & 0.105 & 0.118 \\
$R_{f}=5, R_{c}=5$ & 9.01 & 9.02 & 3.58 & 3.92 \\
$\sigma=0.1$ & & & & \\
$T=30, \alpha=0.2$ & 0.260 & 0.263 & 0.107 & 0.117 \\
$R_{f}=5, R_{c}=5$ & 9.53 & 9.55 & 3.58 & 3.88 \\
$\sigma=0.2$ & & & & \\
$T=30, \alpha=0.2$ & 0.177 & 0.180 & 0.083 & 0.079 \\
$R_{f}=5, R_{c}=5$ & 6.80 & 6.82 & 2.88 & 2.72 \\
$\sigma=-0.1$ & & & & \\
\hline
\end{tabular}


exothermic than the partial oxidation reaction and cause severe temperature increases in the reactor, as well as yielding valueless products. Consequently, any operating policy which enhances the partial oxidation reaction and increases the yield of the desired product will have an impact on productivity and energy usage.

Periodic torcing is implemented by alternating the input concentration of ethylene and/or the total feed flow rate between two prespecified values. A nonsymmetric rectangular pulse $[7-9]$ is used to generate the forcing function (Figure 1). The ratio of maximum to minimum feed concentration, ratio of maximum to minimum feed flow rate, oscillation frequency, oscillation amplitude, duty fraction and phase shift between forcing of different inputs are the parameters studied for enhancing selectivity and yield.

Yield improvements by $17 \%$ have been observed in adiabatic and nonadiabatic forced periodic operation of the ethylene oxidation reactor when both the feed flow rate and feed composition were forced with phase shift (Table 1 , rows 8,10 ). The explosive nature of the ethylene oxide-oxygen mixture imposes a constraint on ethylene feed concentration, and to remain below the explosion limit, ethylene concentration is kept below 2 percent in all our studies. In this region, the steady state profiles do not show any multiplicities. Consequently, large improvements should not be expected. Yet since any improvement obtained is due to periodic variations in valve openings which have negligible operating costs, a yield imp ovement of $10 \%$ or more is quite encouraging. Single variable forcing (Table 1, row 4 - flow rate forcing), and multiple input forcing without phase shift (Table 1, row 6) did not have significant impact on yield. Multiple input forcings where concentration pulse is leading the flow rate pulse (negative phase shift) had an adverse effect on both selectivity and yield (Table 1, rows 11,12). The impact of periodic forcing on the ethylene oxidation reactor operated isothermally was also investigated. Since the reaction rates depend on the reactor temperature through an exponential relationship, little improvement is expected in this mode of operation. However, this was the only mode of operation which could be carried out in the experimental reactor system (Figure 2) utilized for developing the reaction 


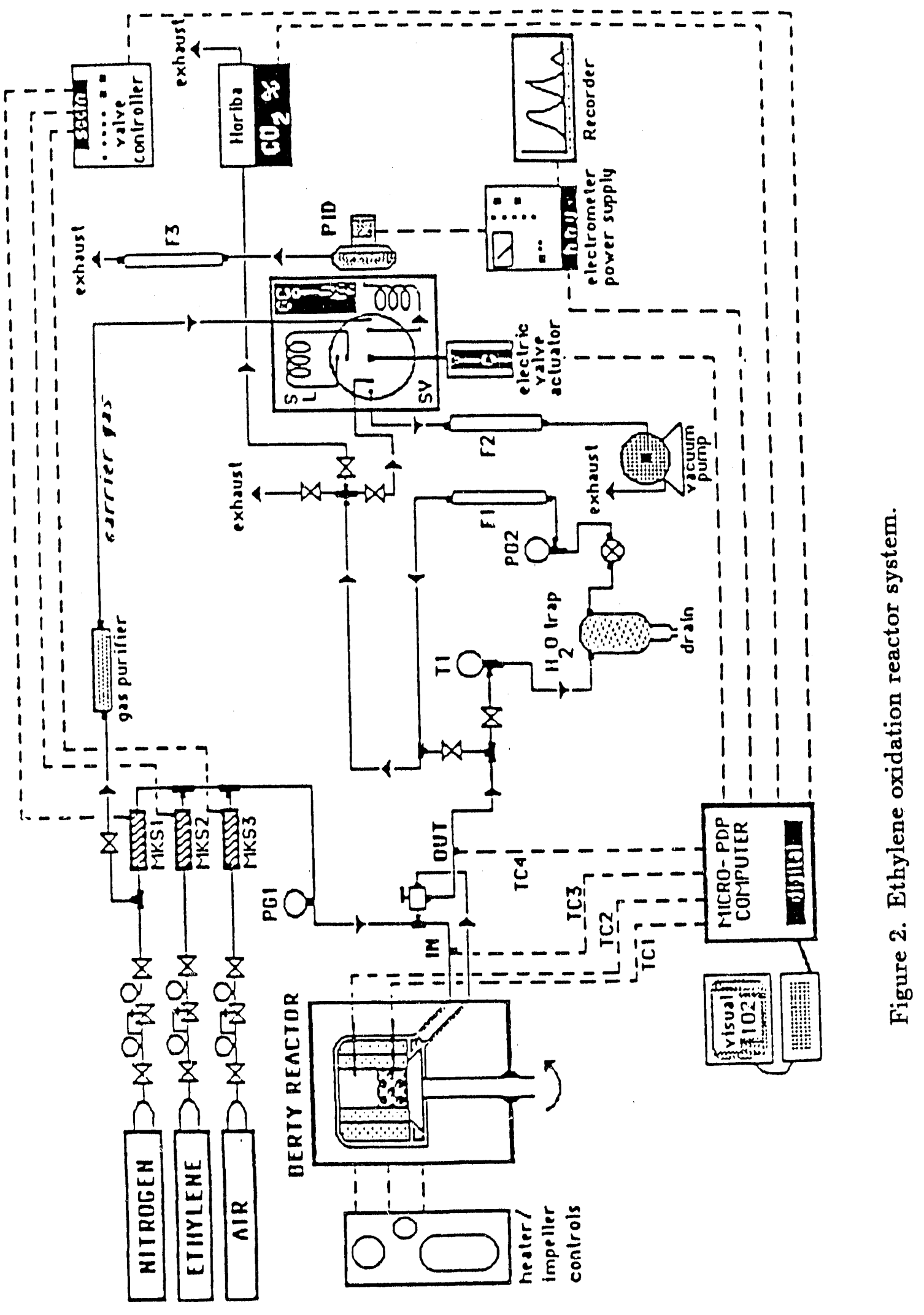


Table 2. Isothermal CSTR $\left(T_{R}=210^{\circ} C, F_{0}=2000 s c c m, C_{E f 0}=1.0 \%\right)$.

\begin{tabular}{lcl}
\hline Parameters & $\begin{array}{l}\text { Vibrational } \\
\text { Control }\end{array}$ & $\begin{array}{l}\text { Experim. } \\
\text { Result }\end{array}$ \\
\hline steady & $\bar{C}=0.16$ & 0.16 \\
state & $\bar{Y}=5.24 \%$ & $5.29 \%$ \\
$T=30, \alpha=0.2$ & 0.16 & 0.16 \\
$R_{f}=7$ & 5.24 & 5.29 \\
$T=30, \alpha=0.2$ & 0.16 & 0.16 \\
$R_{f}=5, R_{c}=5$ & 5.23 & 5.23 \\
$\sigma=0.0$ & & \\
$T=30, \alpha=0.2$ & 0.16 & 0.16 \\
$R_{f}=5, R_{c}=5$ & 5.24 & 5.30 \\
$\sigma=0.1$ & & \\
$T=30, \alpha=0.2$ & 0.16 & 0.16 \\
$R_{f}=5, R_{c}=3$ & 5.26 & 5.40 \\
$\sigma=0.2$ & & \\
$T=30, \alpha=0.2$ & 0.16 & 0.16 \\
$R_{f}=5, R_{c}=5$ & 5.21 & 5.30 \\
$\sigma=-0.1$ & & \\
\hline
\end{tabular}


rate equations, and consequently isothermal operation was used for checking the theoretical results against experimental data. Theoretical and experimental results show good agreement and confirm that there is not much improvement by forced periodic operation of the isothermal reactor (Table 2). Details of the reactor model, the ethylene oxidation reaction rate expressions and the averaged system equations for isothermal, adiabatic and nonadiabatic ethylene oxidation reactor are provided in Appendix 1.

\section{Assessment of Effects of Periodic Forcing by Shooting Algorithm Approach}

Vibrational control method provides an efficient tool for analyzing the forced periodic operation of chemical reactcre. But, two factors limit its widespread use. The derivation of the averaged equations becomes challer.ging as the complexity of the reaction mechanism increases since the use of the method of averaging necessitates the utilization of series expansions. Also, the derivations must be carried out for each specific case separately, causing significant overhead even when syn bolic languages such as Macsyma are used to carry out part of the derivations. Since the industrial research and development groups are more interested in tools that can readily integrate with their process models, we focused on the development of a numerical approach which can ultimately be offered as a general purpose computer program package.

The numerical technique is based on the solution of a boundary value problem by a shooting algorithm [2]. Consider a system of ordinary differential equations of the form

$$
\begin{gathered}
\frac{d \mathbf{x}}{d t}=\mathbf{f}(t, \mathbf{x} ; \mathbf{p}) \\
\mathbf{x}(t=0)=\mathbf{x}_{0} \\
\mathbf{x}, \mathbf{x}_{\mathbf{0}}, \mathbf{f} \in \Re^{n}, \mathbf{p} \in \Re^{m} \text {, and } t \in \Re
\end{gathered}
$$

where $\mathrm{x}$ is the vector of dependent variables, and $\mathrm{p}$ is the vector of forced parameters. Using the state periodicity condition

$$
\mathbf{x}(0)=\mathbf{x}(\tau)
$$


which is satisfied upon convergence to the periodic attractor, a boundary value problem can be formulated. The average value of yield and/or conversion may be computed by calculatirg the values of the appropriate state variables at a fixed number of points on the limit cycle and then by using an averaging equation.

Computational efficiency is improved by solving the differential form of the timeaverage performance measure simultaneously with the syster.. equations. For example, the time-average yield of a system subjected to a periodic input forcing with oscillations of period $\tau$ is defined by

$$
\bar{Y}(\tau) \stackrel{\text { def }}{=} \frac{\frac{1}{\tau} \int_{0}^{\tau} Q_{f}(\theta) \frac{T(\theta)}{T_{f}} C_{E O}(\theta) d \theta}{\frac{1}{\tau} \int_{0}^{\tau} Q_{f}(\theta) C_{E, f}(\theta) d \theta}
$$

Differentiation of (2) with respect to time leads to an additional differential equation which can be appended to the system equations

$$
\frac{d Y}{d t^{\prime}}=\frac{Q_{f} \frac{T}{T_{f}} C_{E O}-Q_{f} C_{E, f} Y}{\int_{0}^{t^{\prime}} Q_{f} C_{E, f} d \theta}-
$$

The correct initial conditions are approximated by iterating

$$
\mathbf{x}-\mathbf{g}(T, \mathbf{x} ; \mathbf{p})=\mathbf{0}
$$

In this equation, $g$ is obtained by integrating Eqn. (1) for one forcing period. The Jacobian of Newton iterations is given by the following $n \times(n+1)$ augmented matrix

$$
\boldsymbol{\Gamma}=\left[\begin{array}{l|l}
\mathbf{I}-\frac{\partial \mathbf{g}}{\partial \mathbf{x}} & -\frac{\partial \mathbf{g}}{\partial p_{d}}
\end{array}\right]=\left[\begin{array}{l|l}
\mathbf{I}-\mathbf{S}(t=T) & \mathbf{P}(t=T)
\end{array}\right]
$$

where I denotes the $n \times n$ unit matrix. The entries of the Jacobian matrix are computed via integration of associated variational equations:

$$
\frac{d \mathbf{S}}{d t}=\left[\frac{\partial \mathbf{f}}{\partial \mathbf{x}}\right] \mathbf{S}(t), \quad \mathbf{S}(t=0)=\mathbf{I}
$$


and

$$
\frac{d \mathbf{P}}{d t}=\left[\frac{\partial \mathbf{f}}{\partial \mathbf{x}}\right] \mathbf{P}(t)+\frac{\partial \mathbf{f}}{\partial p_{d}}, \quad \mathbf{P}(t=0)=\mathbf{0}
$$

To carry out this task, a package which is capable of computing the first order sensitivity coefficients for systems of ODEs. ODESSA [11], is used. When using the method outlined above, the linear stability of the periodic solutions can be determined at no extra cost. Upon convergence, entries of the monodromy matrix (S) are known from the last Newton iteration. A periodic orbit is stable if all of its Floquet multipliers (eigenvalues of the monodromy matrix) lie within the unit circle of complex plane.

For the continuation of periodic solutions, one needs an additional equation:

$$
\sum_{i=1}^{n}\left(\frac{d x_{i}}{d s}\right)^{2}+\left(\frac{d p_{d}}{d s}\right)^{2}=1
$$

Here, $s \in \Re$ is the continuation parameter which approximates the arclength on the solution branch. The resulting system of algebraic equations can be solved by using a predictorcorrector method.

The results obtained by the numerical approach agree well with the results of vibrational control approach (Table 1). Plots of time-average yield as a function of flow rate are shown in Figure 3 and compared with the steady state solutions (solid lines). The ethylene oxide yield is iniproved by periodic forcing of feed concentration and flow rate with phase shift (Fig. 3c), in the whole range of flow rate.

The shooting algorithm program has computational speed advantages and ease of use. Furthermore, it can be incorporated with a nonlinear programming program into an optimization package [12]. This optimization software would enable the selection of the optimal conditions to maximize the yield. The results obtained by the optimization program for the EO reactor are presented in Appendix 2. The optimal values of the duty fraction $(\alpha)$, period $(\tau)$ and phase shift $(\sigma)$ on the average yield are summarized for various ethylene feed concentrations and various feed flow rates (Table 3). Yield improvements as large as $23.6 \%$ have been observed as a result of optimization. 

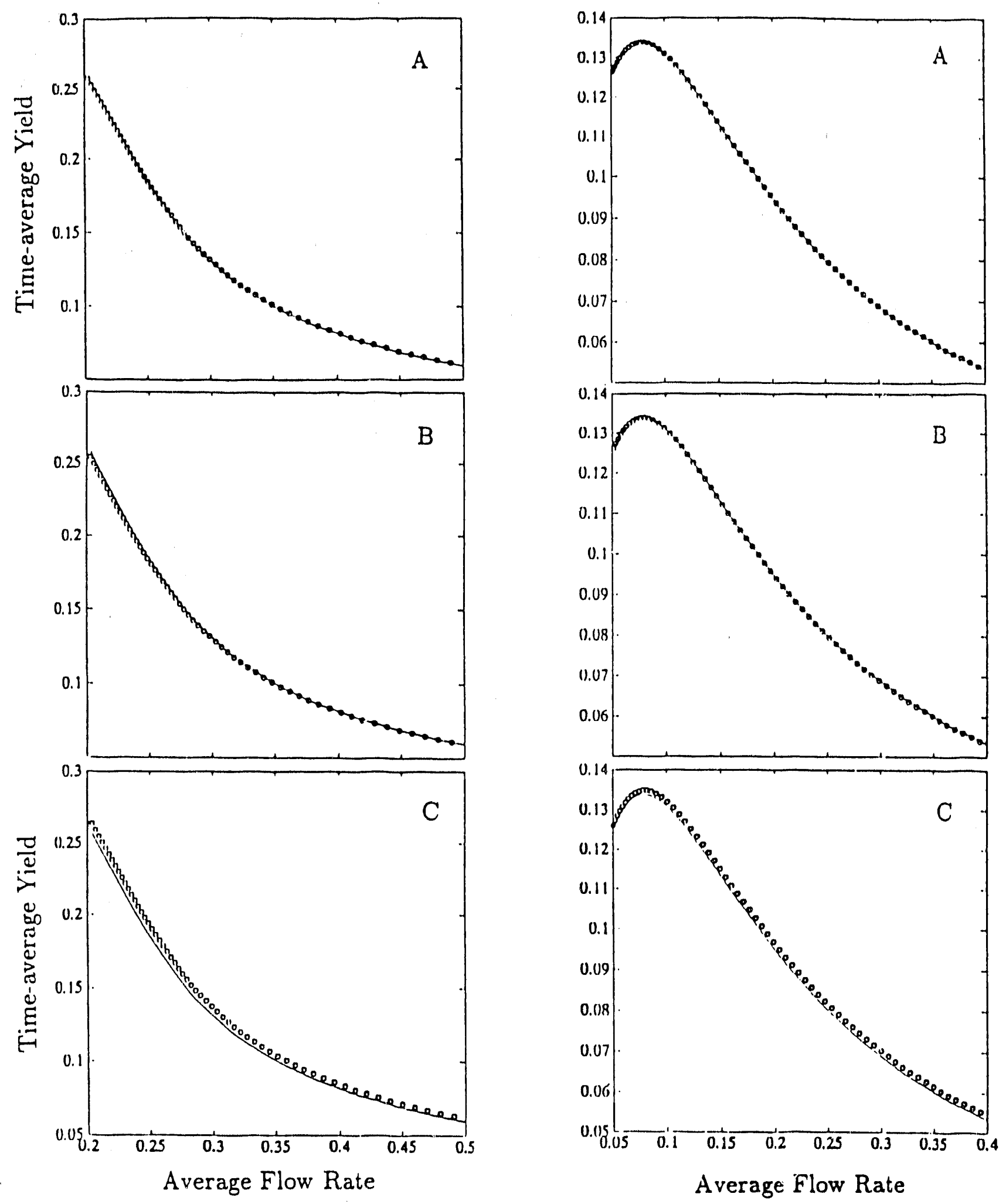

Figure 3. Periodic solutions of the forced CSTR computed by the shooting algorithm. Solid line indicates the steady state solutions. $T=10 \mathrm{sec}, \alpha=0.2$ and $C_{E f 0}=0.35 \%$ (A) Flow rate oscillations, $R_{f}=5$ (B) Multiple oscillations without phase shift, $R_{f}=5, R_{c}=3$ and $\sigma=0.0$ (C) Multiple oscillations with phase shift, $R_{f}=5, R_{c}=3$ and $\sigma=0.1$. 
Table 3. Results of optimization (mean value of ethylene feed concentration, $c_{a v}$, is $1 \mathrm{vol} \%$ ).

\begin{tabular}{lrrrrr}
\hline \hline & $q_{a v}=120$ & $q_{a v}=180$ & $q_{a v}=240$ & $q_{a v}=300$ & $q_{a v}=360 \mathrm{sccm}$. \\
\hline$\alpha^{*}$ & 0.4057 & 0.4024 & 0.4062 & 0.4137 & 0.4225 \\
$\sigma^{*}$ & -0.3561 & -0.3609 & -0.3717 & -0.3854 & -0.3996 \\
$\tau^{\prime *}($ sec. $)$ & 615.31 & 685.81 & 755.61 & 852.81 & 979.22 \\
$Y_{\text {s }} \dagger$ & 11.4946 & 10.7685 & 9.6902 & 8.7044 & 7.8680 \\
$\bar{Y} \ddagger$ & 12.6404 & 12.1843 & 11.2086 & 10.2602 & 9.4319 \\
$\%$ change & 9.97 & 13.15 & 15.67 & 17.87 & 19.88 \\
\hline
\end{tabular}

Results of optimization (mean value of feed flow rate, $q_{a v}$, is $700 \mathrm{sccm}$ ).

\begin{tabular}{lrrrrr}
\hline \hline & $c_{a v}=0.1$ & $c_{a v}=0.2$ & $c_{a v}=0.3$ & $c_{a v}=0.4$ & $c_{a v}=0.5$ vol\% \\
\hline$\alpha^{*}$ & 0.4053 & 0.4148 & 0.4250 & 0.4336 & 0.4411 \\
$\sigma^{*}$ & -0.3671 & -0.3867 & -0.4034 & -0.4164 & -0.4270 \\
$\tau^{\prime *}($ sec. $)$ & 213.73 & 373.21 & 547.42 & 735.31 & 940.18 \\
$Y_{s s} \dagger$ & 9.4635 & 8.2859 & 7.4560 & 6.8542 & 6.3927 \\
$\bar{Y} \ddagger$ & 10.8514 & 9.7824 & 8.9746 & 8.3723 & 7.9015 \\
$\%$ change & 14.67 & 18.06 & 20.37 & 22.15 & 23.60
\end{tabular}

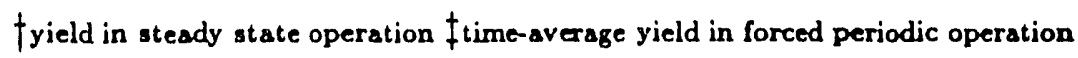


Since the shooting elgorithm and the nonlinear programming software are independent of any reaction or reactor model, the optimization package can be used for assessing the benefis of forced periodic operation and finding the the optimal forcing parameters for any reactor and reaction mechanism. The extension of its use to other types of reaction mechanisms and tubular packed-bed reactor systems are discussed in Chapter V, Conclusions and Future Research Directions.

\section{CLASSIFICATION OF STEADY STATE REACTOR BEHAVIOR BY NUMERICAL SINGULARITY AND BIFURCATION ANALYSIS}

Bifurcation and singularity analysis provide vital information on how some important variables of a reactor are affected by changes in operating conditions. For example, the effects of feed concentration, temperature and flow rate on exit product concentration can be described completely for the ranges of interest in these input variables. This knowledge is essential for selecting the operating conditions that will maximize the desired operation objective while also pointing out the ranges of operating conditions leading to sensitive and dangerous operation of the reactor. The bifurcation diagram provides information on how a dependent variable such as the exit concentration varies with respect to the bifurcation parameter such as the total flow rate. The singularity diagram displays the various regions which have qualitatively different bifurcation behaviors, in the plane of the other two independent (input) variables such as the feed temperature and concentration. A typical scenario in the selection of operating conditions may begin by iıspecting the singularity diagram to pick the range of feed temperature and concentration which provide desirable bifurcation behavior, then construct the bifurcation diagrams to pick the appropriate range of feed flow rate. The singularity and bifurcation diagrams have a significant impact on sensitivity analysis since the effects of variations in input variables can be computed and interpreted efficiently.

Consider a bifurcation diagram (Figure 4) for the tubular packed-bed $\mathrm{CO}$ oxidation 


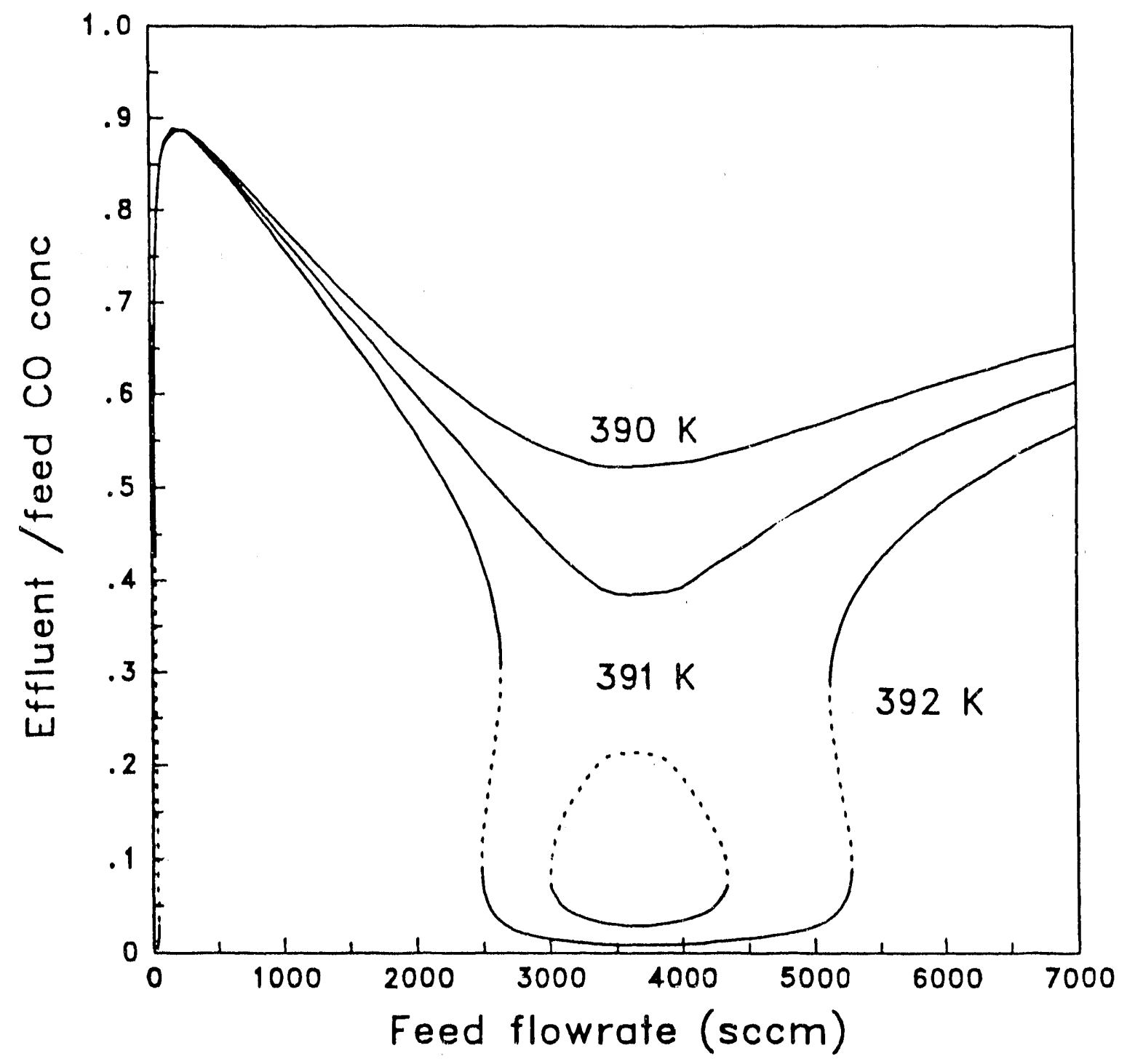

Figure 4. Three different bifurcation behaviors of the autothermal packed-bed tubular CO oxidation reactor when $C_{\text {in }}=0.295$ mole \% CO. 
reactor shown in Figure 5. At a flow rate of $3500 \mathrm{sccm}$, if the feed temperature is $390^{\circ} \mathrm{K}$, the ratio of unreacted $\mathrm{CO}$ (feed) concentration in the effluent stream to $\mathrm{CO}$ inlet concentration is 0.52 and there is a single stable steady state. A $1^{\circ} \mathrm{K}$ increase in feed temperature causes the development of an isolated solution branch, and generates three possible values for the concentration ratio at $0.4,0.22$ (unstable) and 0.03 . Hence a small disturbance may force the system to move from partial to almost total conversion and cause significant temperature increase in the reactor. A second $1^{\circ} \mathrm{K}$ increase in the feed temperature establishes a single stable steady state at a concentration ratio of 0.01 , operation at almost total conversion. In summary, if the feed temperature is set at $390^{\circ} \mathrm{K}$ an increase of $1^{\circ} \mathrm{K}$ in feed temperature combined with another disturbance or a $2^{\circ} \mathrm{K}$ increase would cause severe changes in reactor operation. Generating this type of information by relying only on reactor simulation studies would be quite time consuming and would involve some lucky guesses. Yet the sensitivity of the reactor operated under these conditions to feed temperature variations can be deduced trivially when the singularity diagram is considered (Figure $\epsilon$ ): At 0.295 mole $\% C O$ and dimensionless feed temperature range of 0.917 to 0.923 three regions (I, II, III) of qualitatively different behaviors have been traversed.

The application of singularity theory to bifurcation problems [13] introduced a coherent framework for classifying all of the different behaviors exhibited by a nonlinear system. This has lead to numerous studies of chemical engineering systems where the different bifurcation behaviors were categorized in the remaining parameter space. Analytical techniques have been used in these studies and for simple CSTRs they provide a good approach. Yet these analytical techniques can not be used in systems described by a large number of equations. For example, the steady-state model of the autothermal $\mathrm{CO}$ oxidation reactor (Figure 5) consists of over 100 linear and nonlinear algebraic equations that result from discretizing 7 elliptic partial differential equations [14]. One-point orthogonal collocation was used in the simplification of the radial differential terms and a two-subsection spline collocation scheme was used in the axial direction [15]. The steady-state model after the 


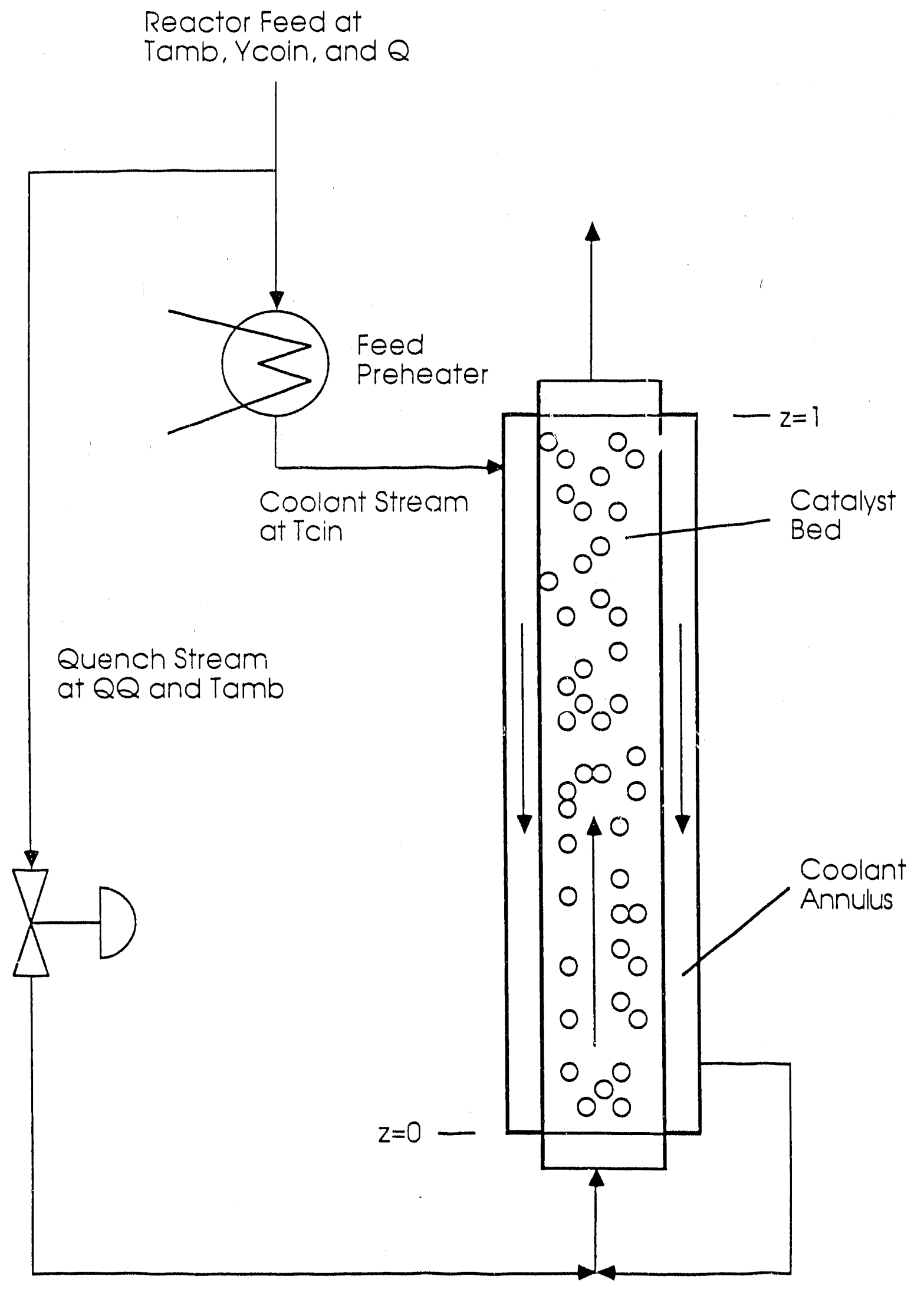

Figure 5. The autothermal tubular CO oxidation reactor system. Quench stream is used in feedback ccntrol studies. 

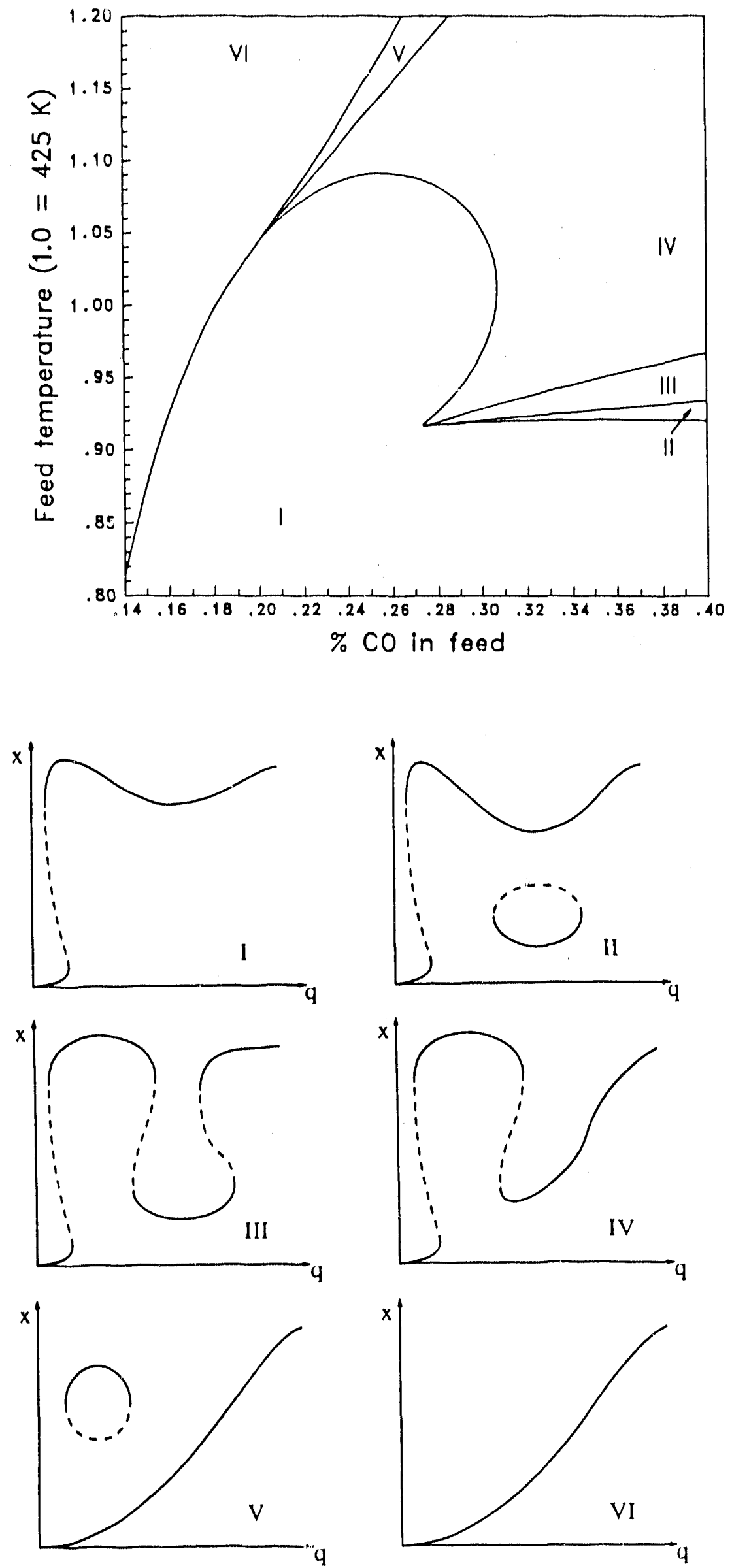

Figure 6. A classification of the different bifurcation behaviors observed for the $\mathrm{CO}$ oxidation reactor. 
simplifications can be represented by

$$
\mathbf{f}(x, \mathbf{y} ; q, t, c)=0
$$

where $\mathrm{f}: \Re^{n} \times \Re^{3} \mapsto \Re^{n},(x)$ is the dependent variable, i.e., the effluent reactant concentration, $(\mathbf{y})$ is the vector of state variables excluding $x,(q)$ is the reactor feed flowrate, $(t)$ is the reactor feed temperature and $(c)$ is the feed reactant concentration. Because $n$ is large and because of the nature of some of the nonlinearities in $f, f$ cannot be reduced explicitly to a scalar. This is a very important point and provided the impetus for developing the numerical techniques that are discussed in the following sections. The numerical singularity analysis technique differs from previous singularity analysis schemes in that the application of the singularity analysis is done numerically and requires no explicit differentiations of the system equations and no reduction of the mathematical model to a scalar equation. Consequently, it is implemented by developing a self contained program that interacts with the program of the reactor model.

\section{Singularity Theory}

Changes in qualitative behavior occur at singular points; the birth of an isolated solution branch or the appearance of hysieresis in a solution branch occurs at a distinct value of $t$ for a given $c$. This means that we are faced with the task of tracing loci of codimension-1 singularities (codimension-2 bifurcations) in the $(t, c)$-plane. By the singularity theory [13], two singularities separate qualitatively different bifurcation behaviors: the simple bifurcation or isola center singularity that satisfies the conditions

$$
\mathbf{f}=\mathbf{0} \quad \text { and } \quad \frac{d q}{d x}=\frac{d x}{d q}=0
$$

and the hysteresis singularity which can be found at

$$
\mathbf{f}=0 \quad \text { and } \quad \frac{d q}{d x}=\frac{d^{2} q}{d x^{2}}=0 .
$$


Translating these conditions into something that can be computed and continued numerically means that solutions to the vector

$$
\mathbf{f}(x, \mathbf{y}, q, t ; c)=\mathbf{0}
$$

must be computed along with

$$
\frac{d \psi}{d x}=\frac{d \psi}{d q}=0
$$

for the isola center and simple bifurcation singularities, and

$$
\frac{d \psi}{d x}=\frac{d^{2} \psi}{d x^{2}}=0
$$

for the hysteresis singularity, where the extra parameter $\psi$ can be $c$ or $t$ in ur reactor model. The equivalence of these conditions ((13) to (10) and (14) to (11)) can be understood by first noting that

$$
\frac{d q}{d x}=\frac{d q}{d \psi} \frac{d \psi}{d x}
$$

This implies that

$$
\frac{d q}{d x}=0 \quad \text { whenever } \quad \frac{d \psi}{d x}=0
$$

if

$$
\frac{d q}{d \psi} \neq 0
$$

We can be assured of (16) holding true when $\psi$ is an extractable parameter. An extractable parameter is a parameter of a nonlinear system that can be uniquely expressed in terms of the other parameters and state variables of the system. It has been shown that the feed temperature $t$ is an extractable parameter for nonadiabatic chemical reactors [15].

Recognition of Codimension-1 Singularities. We take advantage of the extractable nature of $t$ in the following scheme: for some solution $\mathbf{z}_{0}$ of $\mathbf{f}(\mathbf{z})=\mathbf{0}$ where $\mathbf{z}=[\mathbf{y}, t, x, q, c]^{\mathrm{T}}$, the approximate location $\left(x_{1}, q_{1}\right)$ of the singular point can be calculated by

$$
\begin{aligned}
\frac{d t\left(x_{0}, q_{0}\right)}{d x}+\frac{d^{2} t\left(x_{0}, q_{0}\right)}{d x^{2}}\left(x_{1}-x_{0}\right)+\frac{d^{2} t\left(x_{0}, q_{0}\right)}{d q d x}\left(q_{1}-q_{0}\right) & =0 \\
\frac{d t\left(x_{0}, q_{0}\right)}{d q}+\frac{d^{2} t\left(x_{0}, q_{0}\right)}{d x d q}\left(x_{1}-x_{0}\right)+\frac{d^{2} t\left(x_{0}, q_{0}\right)}{d q^{2}}\left(q_{1}-q_{0}\right) & =0 \\
c_{1}-c_{0} & =0
\end{aligned}
$$


for the isola center or simple bifurcation singularity and

$$
\begin{array}{r}
\frac{d t\left(x_{0}, q_{0}\right)}{d x}+\frac{d^{2} t\left(x_{0}, q_{0}\right)}{d x^{2}}\left(x_{1}-x_{0}\right)+\frac{d^{2} t\left(x_{0}, q_{0}\right)}{d q d x}\left(q_{1}-q_{0}\right)=0 \\
\frac{d^{2} t\left(x_{0}, q_{0}\right)}{d x^{2}}+\frac{d^{3} t\left(x_{0}, q_{0}\right)}{d x^{3}}\left(x_{1}-x_{0}\right)+\frac{d^{3} t\left(x_{0}, q_{0}\right)}{d q d x^{2}}\left(q_{1}-q_{0}\right)=0 \\
c_{1}-c_{0}=0
\end{array}
$$

for the hysteresis singularity. Note that these three conditions imply that $c$ is fixed at a constant value in this step of the continuation scheme. These pairs of Taylor series expansions give an estimate of the location of the singularity in the $(x, q)$-plane provided that $\left(x_{0}, q_{0}\right)$ is sufficiently close to the correct value. We continue to the new estimate of the singular point by first predicting the values of $\mathbf{z}_{1}$ by

$$
\overline{\mathbf{z}}_{1}=\mathbf{z}_{0}+\frac{d \mathbf{z}}{d s} \delta s
$$

where

$$
\delta s=\sqrt{\left(x_{1}-x_{0}\right)^{2}+\left(q_{1}-q_{0}\right)^{2}}
$$

and where the vector of derivatives is given by

$$
\left(\begin{array}{cccc}
\partial \mathbf{f} / \partial \mathbf{r} & \partial \mathbf{f} / \partial x & \partial \mathbf{f} / \partial q & \partial \mathbf{f} / \partial c \\
\mathbf{0} & 1 & 0 & 0 \\
0 & 0 & 1 & 0 \\
\mathbf{0} & 0 & 0 & 1
\end{array}\right) \frac{d \mathbf{z}}{d s}=\left(\begin{array}{c}
\mathbf{0} \\
\left(x_{1}-x_{0}\right) / \delta s \\
\left(q_{1}-q_{0}\right) / \delta s \\
0
\end{array}\right)
$$

where $\mathbf{r}=[\mathbf{y}, t]^{\mathrm{T}}$. A Newton-Raphson corrector step completes this straightforward continuation scheme. In the Newton step, $x, q$, and $c$ act as a fixed parameters and $t$ and $\mathbf{y}$ is allowed to float. Note that arc-length parameterization [16] is not needed when $t$ is an extractable parameter.

Now that a solution that is closer to the singular point has been computed, the derivatives of equations (17) or (18) (depending on the singularity sought) need to be updated. Since the discretization of the PDEs that make up the tubular reactor model leads to a very large number of algebraic equations defining $\mathbf{f}$ ( $\mathbf{f}$ is defined in (9) and 
contains $>100$ equations), direct evaluation of the required derivatives promised to be a most egregious task, even with Macsyma or other mathematical packages. We chose to compute the derivatives numerically with a finite-difference scheme. By computing the mesh of solution points seen in Figure 7a for a fixed value of $c$, the required derivatives were easily and accurately calculated.

Continuation of Codimension-1 Singularities. A brute-force scheme of incrementally changing $c$ and applying the algorithm described in the previous section would work in computing the singularity loci, but would be inefficient and would be unable to continue past turning points in the singularity loci. Both of these problems can be eliminated by using a quadratic predictor step in the overall singularity continuation scheme. This idea can be implemented after the first three points in a singularity locus have been computed (three points representing three slightly different values of $c$ ). Our scheme can be understood by considering Figure $7 \mathrm{~b}$ and the following expression:

$$
\mathbf{z}(\tau=\delta \tau)=\mathbf{z}(0)+\left[\frac{d \mathbf{z}}{d \tau}+\left(\frac{1}{2} \delta \tau_{1}\right) \frac{d^{2} \mathbf{z}}{d \tau^{2}}\right] \delta \tau+\frac{1}{2} \frac{d^{2} \mathbf{z}}{d \tau^{2}}(\delta \tau)^{2}
$$

and where

$$
\begin{gathered}
\delta \tau_{j}=\sqrt{\left(x_{j}-x_{j+1}\right)^{2}+\left(q_{j}-q_{j+1}\right)^{2}+\left(t_{j}-t_{j+1}\right)^{2}+\left(c_{j}-c_{j+1}\right)^{2}} \quad j=1,2 \\
\frac{d \mathbf{z}}{d \tau}=\frac{\left(\mathbf{z}_{1}-\mathbf{z}_{2}\right)}{\delta \tau_{1}}, \quad \text { and } \quad \frac{d^{2} \mathbf{z}}{d \tau^{2}}=\frac{\left(\mathbf{z}_{1}-\mathbf{z}_{2}\right) / \delta \tau_{1}-\left(\mathbf{z}_{2}-\mathbf{z}_{3}\right) / \delta \tau_{2}}{\left(\delta \tau_{1}+\delta \tau_{2}\right) / 2}
\end{gathered}
$$

The extra term, i.e., the

$$
\left(\frac{1}{2} \delta \tau_{1}\right) \frac{d^{2} z}{d \tau^{2}}
$$

in what otherwise is a straightforward Taylor series expansion is used to shift the location of the point at which the first derivative is evaluated up to $\tau=0$. This correction is necessary because $d \mathbf{z} / d \tau$ is valid only at $\tau=-\delta \tau_{1} / 2$ and not at the required point $\tau=0$. Higherorder corrections are obviously possible, but were found to be unnecessary when our scheme 


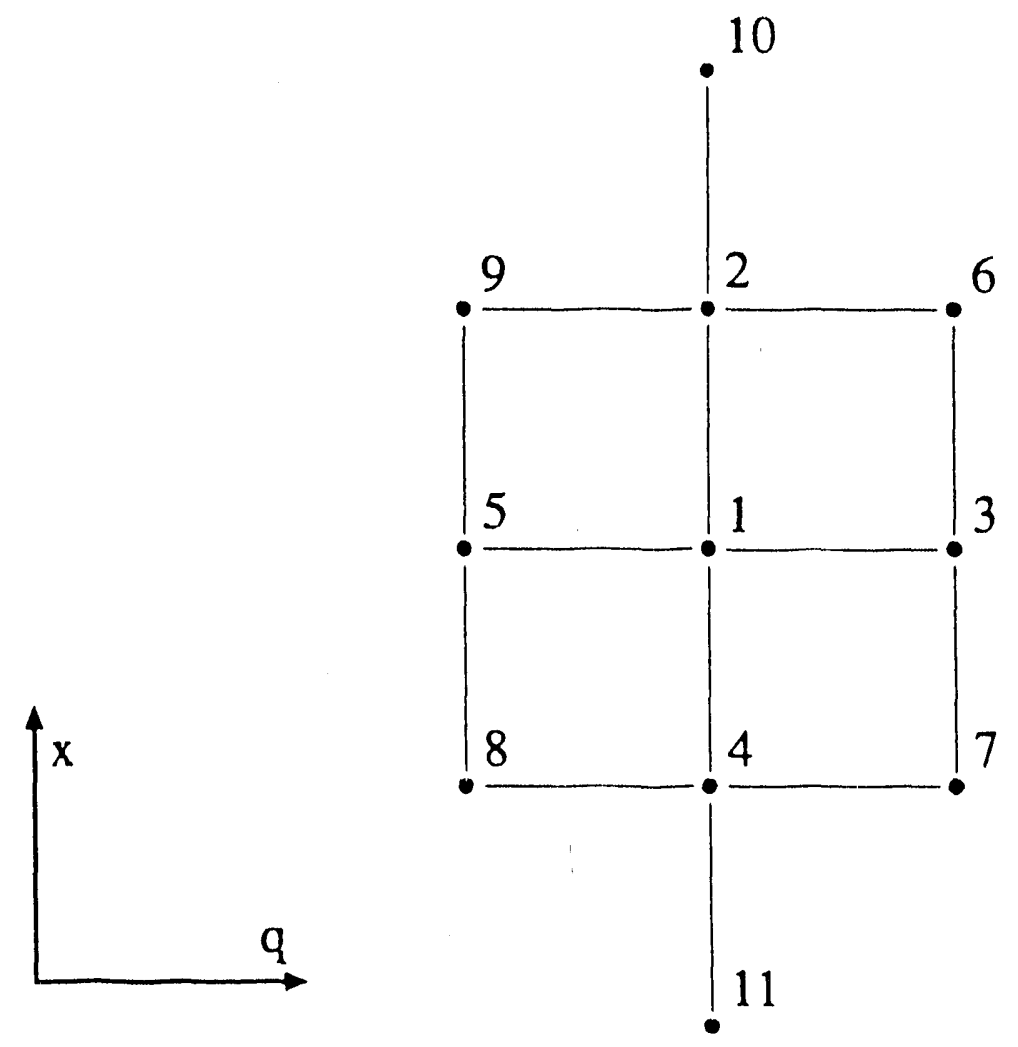

a

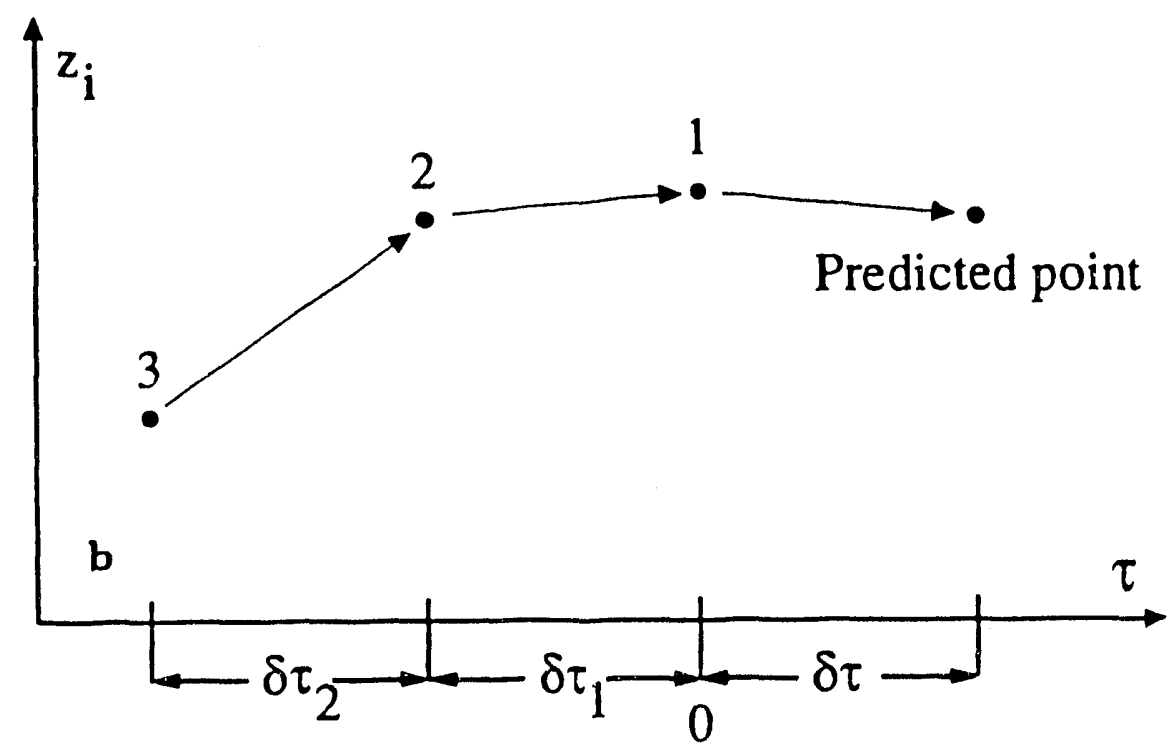

Figure 7. (a) The node net used to compute the derivatives required in finding codimension1 singularities. (b) Predicting the next singular point. 
was used. The reasons for using the quadratic predictor instead of a reparameterization (through arc-length or by a dependent variable exchange) are discussed in Appendix 3.

\section{Computational Details}

A 2500 line FORTRAN program named biff has been written to do both the solution surface computation and numerical singularity analysis tasks. The program is set up in a structure similar to an ODE solver-the user needs only to change the subroutine that contains the model and sections in the main program that pertain to problem-specific I/O demands and the like. All of the results presented in this paper have been computed by biff.

The computation of the points in the net of nodes used in the singularity analysis are independent tasks that can be executed simultaneously after the center node is established. This makes the parallelization of biff simple since only one main do-loop is involved. Taking advantage of parallel processing $[17]$ is important since a single singularity locus of Figure 6 takes about $6 \mathrm{CPU}$ hours to compute and a manifold can take roughly $2 \mathrm{CPU}$ weeks to construct in 1 VUPS (VAX unit of performance) computers. In the parallelized mode, the computation time of a singularity locus can be reduced by a factor of 3 . These figures were obtained from the analysis of the tubular reactor system which contained 123 state variables after discretization of the PDEs. The computer used was a six processor Encore Multimax with a floating-point accelerator. Each processor on this machine is capable of 2 MIPS ( $\simeq 2$ VUPS). All of the arithmetic was done in double precision.

\section{Bifurcation Behavior of Feedback Controlled Reactors}

The traditional approach in assessing the behavior of feedback controlled chemical reactor systems is to conduct a number of simulation studies in the neighborhood of a desirable operating point. Often it is hoped that other centers of attraction do not exist in the vicinity of the region investigated and that the reactor system will be operated such that it will stay within the bounds of the region investigated. However, the $r$ gions 
selected may be too small due to severe nonlinearities in most chemical reactor systems. This would put a heavy responsibility on the control system which must keep the reactor operation within the region selected. Furthermore, simulation studies do not yield reliable information about qualitatively different reactor behavior which might result due to small changes in the operating conditions caused by disturbances. Since the region outside of the immediate neighborhood of the nominal operating conditions becomes open to inspection in bifurcation diagrams, such things as whether the setpoint is globally unique and what should be expected in the event of controller saturation can be investigated.

These points can be illustrated by considering Figure 8 where a state variable denoted as $x$ is plotted as a function of $Q$, a parameter of the physical system in question. The state variable $x$ might be the concentration of reactant in the reactor effluent stream and the parameter $Q$ could be the flowrate of reactants to the reactor-a parameter which will vary quasi-statically from day to day depending on the demand for the product produced in the reactor. Other important parameters such as feed temperature and reactant feed concentration are held constant. In this bifurcation diagram, one can see that the stabilized state is unique for values of $Q$ only in the interval $Q^{-}<Q<Q^{+}$. Other interesting features of this system are the bifurcation points created by the saturation of the controller at each end of the stabilized segment. While the subcritical bifurcation (a) is undesirable, excursions beyond $Q>Q^{++}$will only result in the reactor extinguishing to its lower steady-state, and operation at the desired state can be regained by simply lowering the flowrate to $Q<Q^{+}$. The supercritical bifurcation (b), on the other hand, is an entirely different matter. Lowering the flowrate to values below $Q<Q^{--}$places the reactor in a region where the only stable attractor is the upper steady-state, a state where undesirable products might be created or where the reactor might incur damage due to the heat produced by the increased rate of an exothermic reaction. To add to this dangerous situation, the value of $Q^{-}-Q^{--}$might be so great that the reactor would have to be shut down in order to restore it to the desired state. Thus, we can see how some bifurcations 


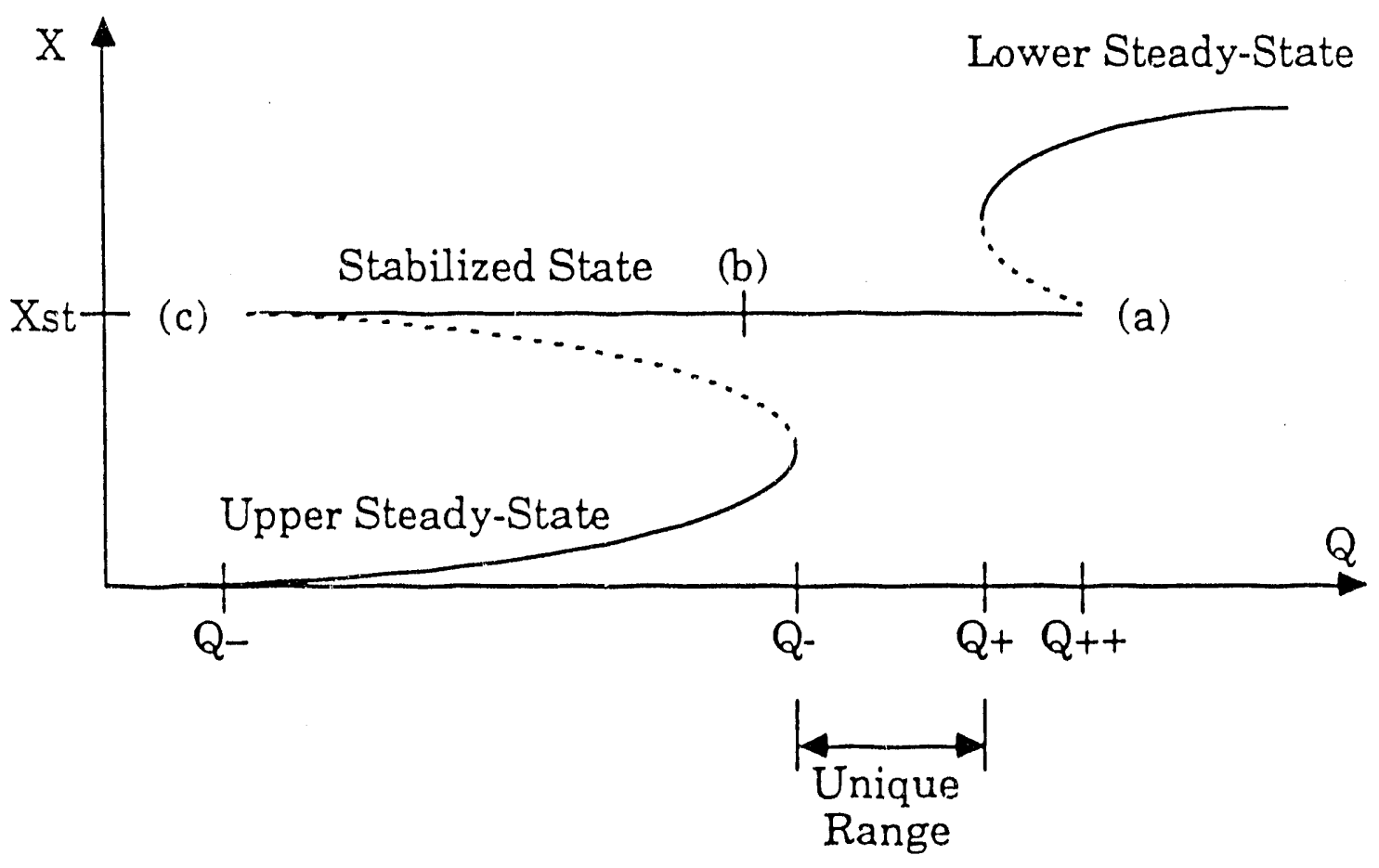
(a) $Q Q=0 \quad$ (Safe bifurcation)
(b) $Q Q=Q Q_{s} \quad$ (Nominal conditions)
(c) $Q Q=2 Q Q_{s} \quad$ (Dangerous bifurcation)

Figure 8. A sample bifurcation diagram. The dashed lines indicate unstable steady states. 
are undesirable and some can be categorized as very undesirable or downright dangerous.

The mathematical model of the tubular packed-bed $\mathrm{CO}$ oxidation reactor is used in the application of the numerical bifurcation and singularity analysis techniques developed to feedback controlled reactors. The reactor system and the hardware for implementing feedback control are illustrated in Figure 2. Two types of control actions, proportional (P) and proportional-integral (PI) control are considered. The controller adjusts the quench flowrate according to the deviation from the desired effluent $\mathrm{CO}$ conversion setpoint

$$
Q Q=Q Q s+k\left(X_{s t}-X_{b N}\right)+k^{\prime} \int_{0}^{t}\left(X_{s t}-X_{b N}\right) d \tau
$$

where $X_{b N}$ is the effluent CO concentration, $Q Q$ s is the nominal quench gas flow, $k$ is the proportional gain and $k^{\prime}$ is the integral "gain" which is set equal to zero for P control only. $Q Q$ is taken to be bounded in the interval $Q Q \in[0,2 Q Q$.$] .$

Behavior Modification by Feedback Control. The effect of feedback control on the bifurcation behavior of a chemical reactor can be considerable (Figure 9a). The most prominent change is the creation of new equilibrium states where there were none which results in the possibility of 5 steady-states for a single value of $Q$. (The open-loop system admits at most three equilibrium states when the feed concentration of $\mathrm{CO}$ is less than 1.4\%.) Benefits of this type of analysis include the global understanding of the system being analyzed which shows what surprises can lurk outside the neighborhood of the setpoint (namely turning points) or how a small change in another parameter (i.e., $T_{\text {cin }}$ ) can drastically affect the range in which the system remains stable for changes in the nominal value of the bifurcation parameter. This last quality can be appreciated by comparing the different lengths of stabilized solution arc (the branches hovering about $X_{b N}=0.5$ ) when $T_{\text {cin }}=424 \mathrm{~K}$ (longer arc) with the shrrter arc generated when $T_{\text {cin }}=416 \mathrm{~K}$. The slight bend in the the stabilized solution branches is a result of the offset inherent in proportional controllers. As expected, under PI control (Fig. 9b) the offset and consequently the bend are eliminated. 

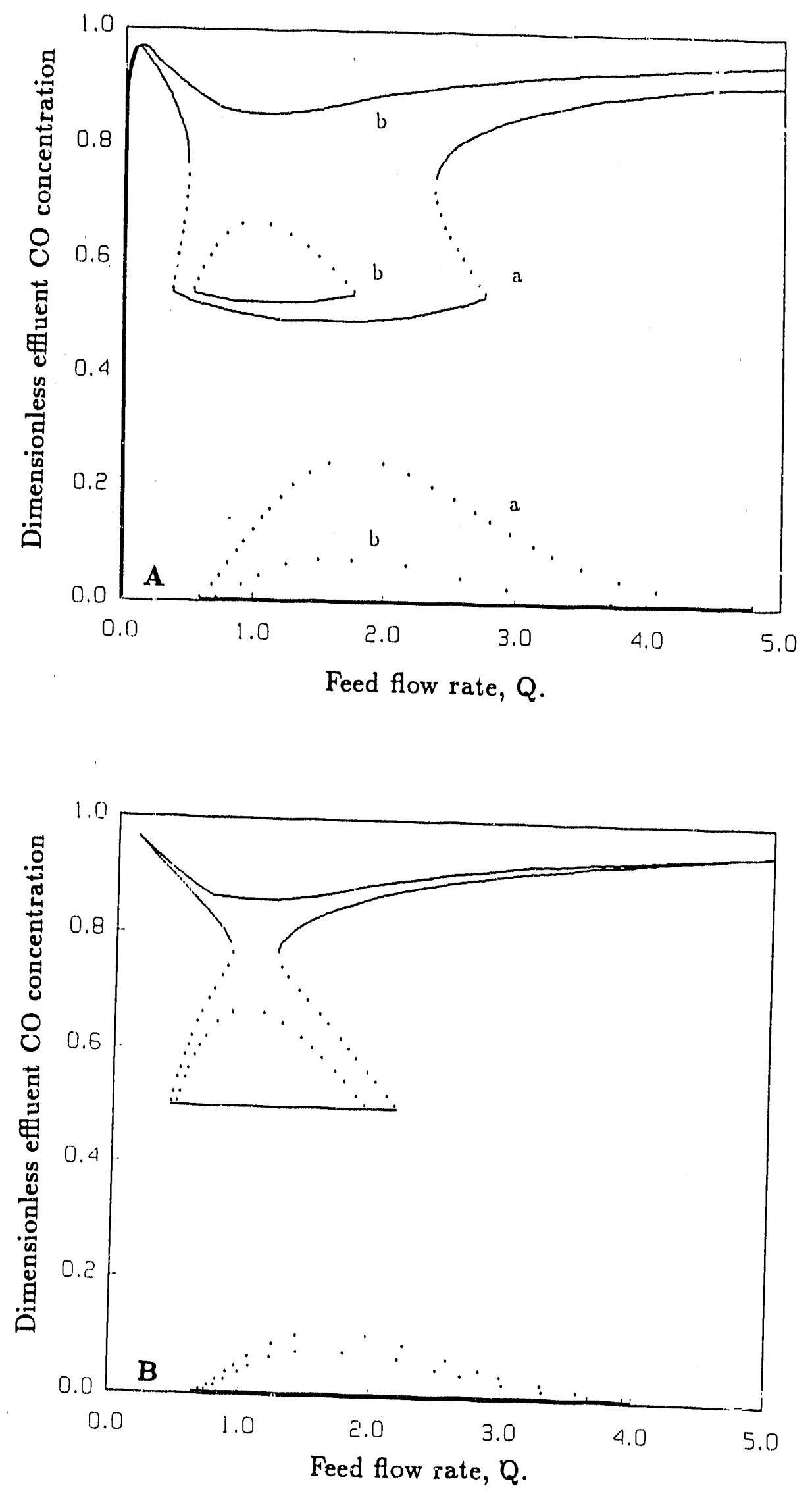

Figure 9. Bifurcation diag ams for the feedback controlled reactor. $X, t=0.5, Q Q \_=0.02$, $1 \% \mathrm{CO}$ in the feed stream. (A) P control, (B) PI control. 
The classification of the different multiplicity patterns in $\left(T_{c i n}, C_{i n}\right)$-space is presented in Figure 10. The curves generated from the organizing center " $A$ " in the figure are those resulting from the open-loop system. The other set of curves traced by solid lines are due to $P$ control while the broken lines represent the effect of PI control. Detailed studies have been conducted for the higher values of feed concentration $\left(C_{i n}>0.35 \% \mathrm{CO}\right)$. The addition of feedback control to the reactor system dramatically alters the bifurcation picture, as is evident by the additional equilibrium states, the increased complexity of the classification diagram, and the appearance of multiple isolas. As a result of the controller, the number of different bifurcation behaviors exhibited by the reactor has been increased from four in the open loop reactor system to thirteen in the feedback-controlled system.

Returning to the issue of safe and dangerous bifurcations, Figure 10 indicates the regions of $\left(T_{c i n}, C_{i n}\right)$-space where dangerous behavior exists. Bifurcation behaviors $\mathrm{V}$ through IX can be classified as dangerous by the criteria set forth in the Introduction. On the other hand, behaviors IV and XIII are safe, but are nonunique and since the stabilized state lies on an isola changes in $Q$ cannot be used to return the system to the desired state if a turning point is crossed. Behavior $\mathrm{X}$ does not contain a stabilized state and behavior I may contain one only at very low feed $\mathrm{CO}$ concentration and high $T_{\text {cin }}$. Behaviors XI and XII are safe, but may not possess a range of $Q$ where the stabilized state is unique. Behaviors II and III are safe and both possess a range of $Q$ where the desired state is unique, so inspection of Figure 10 will reveal where the reactor should be operated to take advantage of this behavior.

\section{Bifurcation Behavior of Periodically Forced Reactors}

Preliminary studies in extending these techniques to analyze the behavior of reactors with forced periodic inputs gave promising results. The loci describing the location of the turning and inflection points of a nonadiabatic CSTR as a function of forcing the inlet flow rate $\mathrm{Q}$ at a period $\mathrm{T}$ are shown in Figure 11. The dotted lines indicate the static 

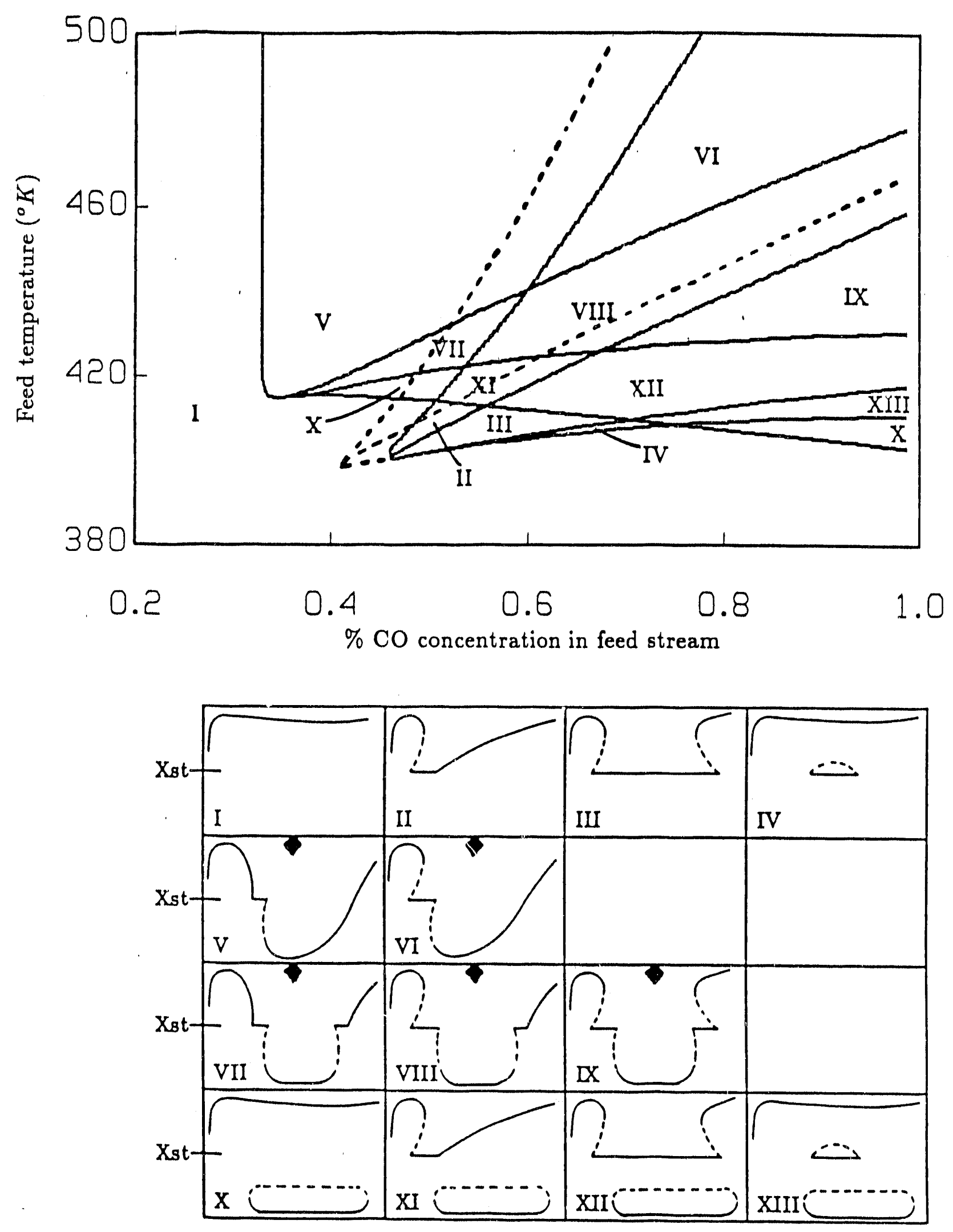

Denotes
Undesirable
Behavior

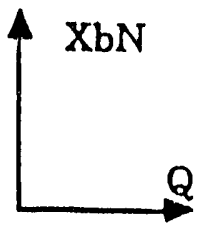

Figure 10. A classification of the different bifurcation behaviors exhibited by the CO oxidation reactor under feedback control, in $\left(T_{\text {cin }}, C_{i n}\right)$-space. 


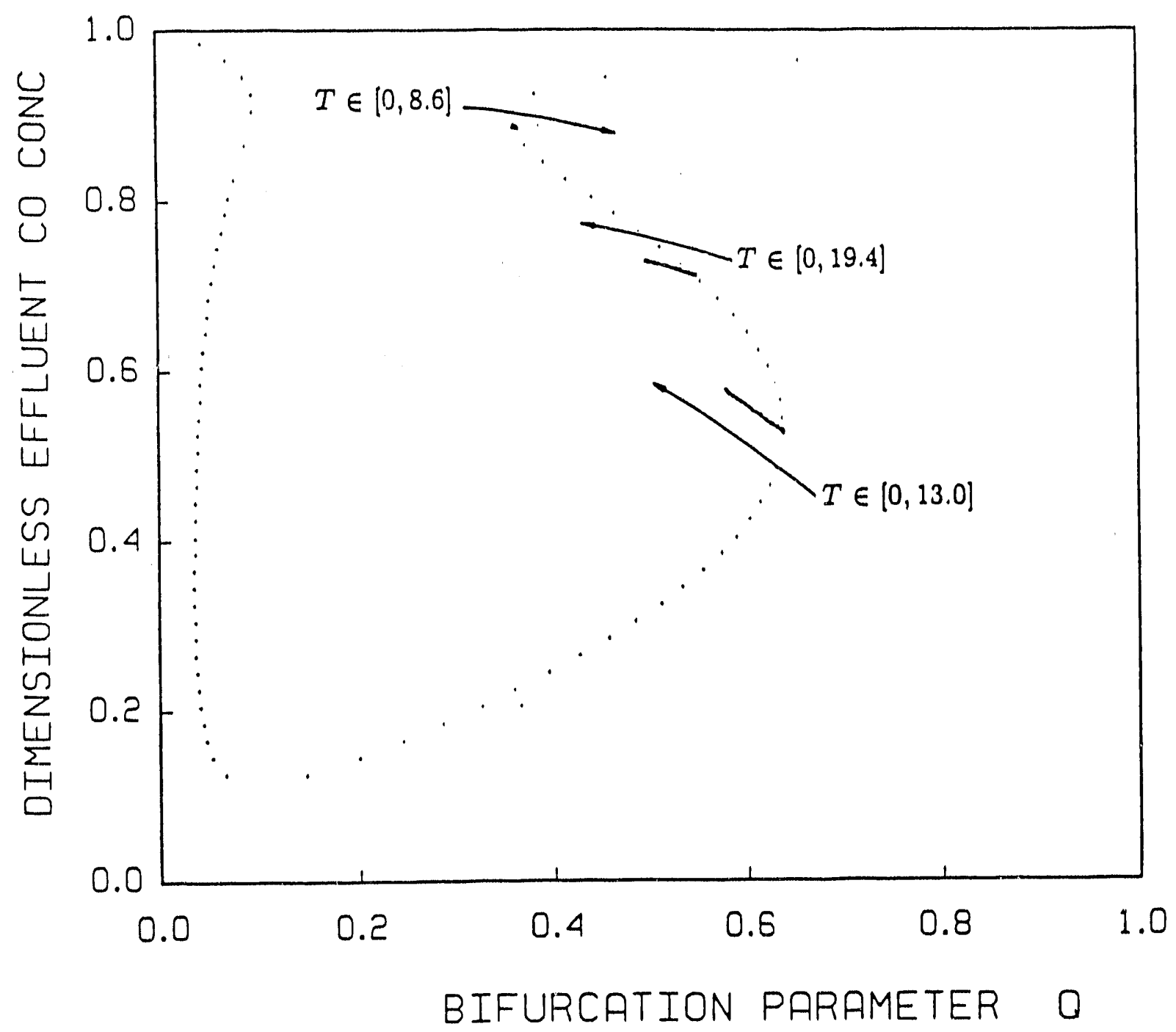

Figure 11. Turning and Inflection Point Locations as a Function of Forcing Function Period in the Nonadiabatic CSTR. $\alpha=25, \beta=75, \omega=0.05, v_{c}=0.5$, $\mathcal{A}_{3}=10$, and $v_{\text {in }}=1.4$. 
equilibrium locus for the CSTR without forcing and the arrows indicate the directions taken by the turning and inflection points as a function of increasing $\mathrm{T}(\mathrm{T}=0$ denotes steady state operation). Periodic forcing of the total reactant flow rate $Q$ has reduced the range of $Q$ having multiple steady states and unstable steady states.

This analysis scheme is based on the discretization of limit cycles resulting from periodic operation by orthogonal collocation and it allows the use of the numerical bifurcation and singularity analysis tools developed. Utilization of orthogonal collocation to discretize the limit cycles provided numerical results that were dependent on the shape of the limit cycle and the accuracy of the results decreased as the limit cycles became elongated. Consequently, our current effort is focused on the use of the shooting algorithm approach described in Chapter II for limit cycle computation. This new approach would provide the building blocks for extending the technique to tubular reactors.

\section{VIBRATIONAL-FEEDBACK CONTROL OF CHEMICAL REACTORS}

Advantages of periodically time varying control over time invariant control have been discussed in recent literature. Time invariant feedback control can not alter the location of system zeros. The improvements due to time varying feedback controllers have been attributed to their zero placement capabilities [19]. There are many systems in the chemical process industries which have undesirable system zero locations. In particular, an odd number of right half-plane zeros indicate systems with inverse response behavior. Tubular packed-bed reactors are among process equipment which may have inverse behavior due to difference of travel times of concentration and temperature waves through the reactor. Systems with inverse response behavior pose challenging control problems, since the initial control action will be in the wrong direction as well. Aside from the deterioration of closed-loop performance, other serious effects can also occur if the system is nonlinear and displays multiple steady states in the region of operation. In the case of packed-bed tubular reactors, inverse response may cause ignition or extinction of the reactor. Consequently, 
it is of interest to assess whether the zero placement capabilities of the periodic feedback controllers can offer any improvement of the dynamic behavior over time invariant feedback.

This study focused on three issues: (1) The assessment of gain margin increase; (2) The evaluation of effects on dynamic system behavior; (3) The development of a vibrationalfeedback controller tuning methodology. The approach proposed by Lee, Meerkov and Runolfsson [19] has been adopted as the method of analysis and their controller design methodology has been extended. A reduced-order linear model of the $\mathrm{CO}$ oxidation reactor has been used as a test case.

\section{Vibrational-Feedback Control Method}

The Vibrational-Feedback Controller. Consider the time-invariant SISO plant

$$
\begin{aligned}
& \dot{\mathbf{x}}=\mathbf{A} \mathbf{x}+\mathbf{b} u \\
& y=\mathbf{C x}
\end{aligned}
$$

where $\mathbf{C A}^{k-1} \mathbf{B} \neq 0$ for $k=1, \quad$ and $\quad \mathbf{x} \in \Re^{n}$. Consider a periodic output controller

$$
\begin{aligned}
\dot{\mathbf{z}} & =\left[\mathbf{F}+\frac{1}{\epsilon} \mathbf{F}_{1}\left(\frac{t}{\epsilon}\right)\right] \mathbf{z}+\mathbf{G} y \\
u & =\left[\mathbf{H}+\frac{1}{\epsilon} \mathbf{H}_{1}\left(\frac{t}{\epsilon}\right)\right] \mathbf{z}
\end{aligned}
$$

where $0<\epsilon \ll 1 \quad$ and $\quad z \in \Re^{n}$, with

$$
\begin{aligned}
& \mathbf{F}=\left(\begin{array}{cccc}
0 & 1 & \ldots & 0 \\
\vdots & \vdots & \ddots & \vdots \\
0 & 0 & \ldots & 1 \\
f_{1} & f_{2} & \ldots & f_{n}
\end{array}\right) \\
& \mathbf{F}_{1}\left(\frac{t}{\epsilon}\right)=\left(\begin{array}{ccc}
0 & \ldots & 0 \\
\vdots & \ddots & \vdots \\
0 & \ldots & \alpha\left(\frac{t}{\epsilon}\right)
\end{array}\right) \\
& \mathbf{G}=\left(\begin{array}{llll}
0 & 0 & \cdots & 1
\end{array}\right)^{T} \\
& \mathbf{H}=\left(\begin{array}{llll}
h_{1} & h_{2} & \ldots & h_{n}
\end{array}\right) \\
& \mathbf{H}_{1}\left(\frac{t}{\epsilon}\right)=\left(\begin{array}{llll}
\beta_{1}\left(\frac{t}{\epsilon}\right) & \cdots & \beta_{n-1}\left(\frac{t}{\epsilon}\right) & 0
\end{array}\right)
\end{aligned}
$$


Let $\tau=t / \epsilon . \quad$ A set of suitable functions $\alpha(\tau)$ and $\beta_{t}(\tau)$ are $\alpha(\tau)=\alpha \cos (\tau)$ and $\beta_{i}(\tau)=h_{i}^{(1)} \cos (\tau)$.

The Averaged Closed Loop System. The closed-loop equation of a system consisting of plant (23) and controller (24) is:

$$
\left(\begin{array}{c}
\dot{\mathbf{x}} \\
\dot{\mathbf{z}}
\end{array}\right)=\left(\begin{array}{cc}
\mathbf{A} & \mathbf{B H}+\frac{1}{\mathfrak{G}} \mathrm{BH}_{1}(\tau) \\
\mathbf{G C} & \mathbf{F}+\frac{1}{\epsilon} \mathbf{F}_{1}(\tau)
\end{array}\right)\left(\begin{array}{l}
\mathbf{x} \\
\mathbf{z}
\end{array}\right)
$$

Let

$$
\left(\begin{array}{l}
\mathbf{x} \\
\mathbf{z}
\end{array}\right)=\left(\begin{array}{cc}
\mathbf{I} & \mathbf{M}(\tau) \\
0 & \mathbf{\Phi}(\tau)
\end{array}\right)\left(\begin{array}{l}
\theta \\
\phi
\end{array}\right)
$$

where

$$
\left(\begin{array}{ll}
\mathbf{I} & \mathbf{M}(\tau) \\
0 & \mathbf{\Phi}(\tau)
\end{array}\right)
$$

is a fundamental matrix for

$$
\left(\begin{array}{cc}
0 & \frac{1}{6} \mathrm{BH}_{1}(\tau) \\
0 & \frac{1}{\epsilon} \mathrm{F}_{1}(\tau)
\end{array}\right)
$$

As a result of the substitution for $(\mathbf{x} z)^{T}$ Eq. (26) reduces to an equation in the standard form in time $\tau=t / \epsilon$ :

$$
\left(\begin{array}{c}
\dot{\theta} \\
\dot{\phi}
\end{array}\right)=\left(\begin{array}{cc}
\mathrm{A} & \mathrm{AM}+\mathrm{BH} \Phi-\mathrm{M} \Phi^{-1} \mathbf{F} \Phi \\
\Phi^{-1} \mathrm{GC} & \boldsymbol{\Phi}^{-1} \mathrm{GCM}+\boldsymbol{\Phi}^{-1} \mathbf{F} \boldsymbol{\Phi}
\end{array}\right)\left(\begin{array}{c}
\theta \\
\phi
\end{array}\right)
$$

Therefore, by applying the averaging principle and by taking into account the conditions imposed, an asymptotic approximation to Eq. (27) can be obtained:

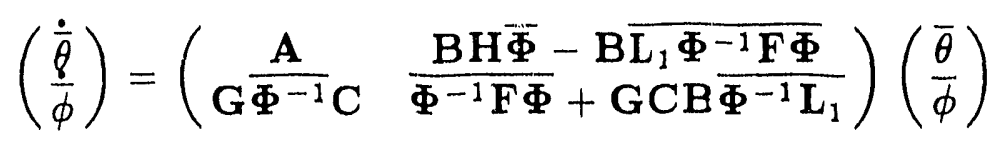

Eq. (28) can be written as a unity feedback closed-loop system (Fig. 12):

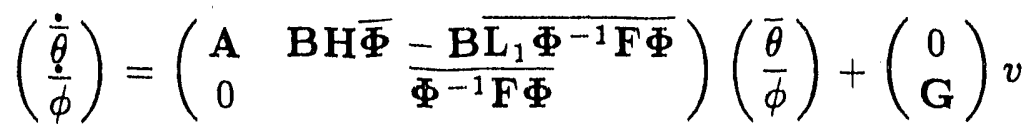

$$
\begin{aligned}
& w=\left(\overline{\Phi^{-}} \mathrm{C} \quad \mathrm{CB} \overline{\Phi^{-1} \mathrm{~L}_{1}}\right)\left(\frac{\bar{\theta}}{\bar{\phi}}\right) \\
& v=w
\end{aligned}
$$




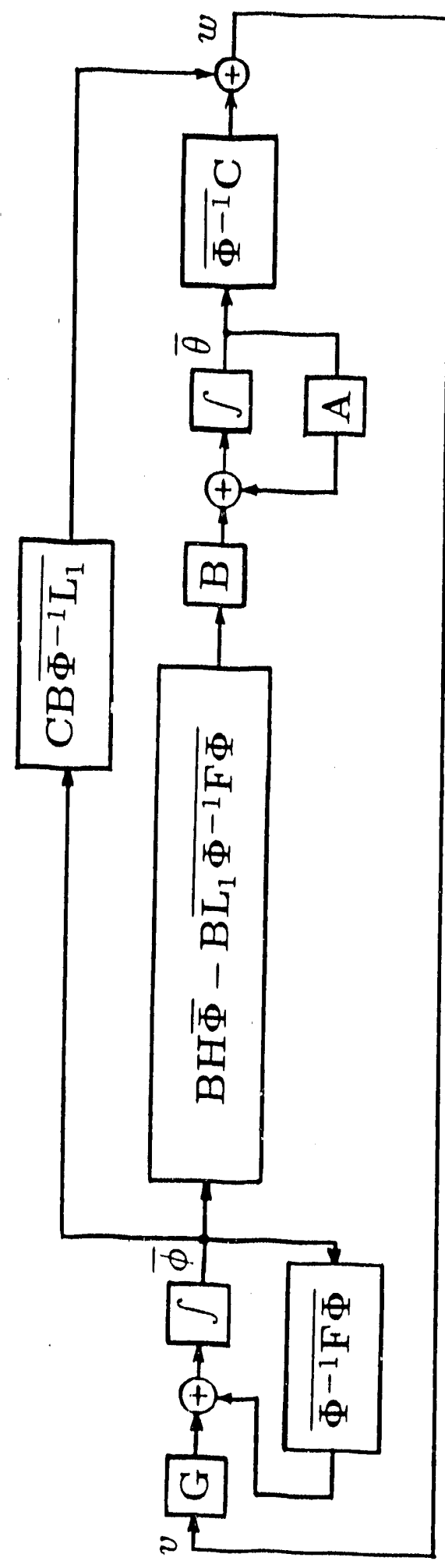

D 
where $\mathbf{L}_{1}=\int \mathbf{H}_{1}(\tau) d \tau$. All finite OL zeros of system (29) are arbitrarily assignable iff $(\mathbf{A}, \mathbf{B}, \mathbf{C})$ of (1) is controllable and assignable. Note that $\mathrm{n}$ open loop poles of (29) coincide with the eigenvalues of $\mathbf{A}$ and the remaining $\mathrm{n}$ open loop poles can be placed by an appropriate selection of $\mathbf{F}$ and $\alpha(\tau)$. The open loop zeros can be placed by proper selection of $h_{i}$ and $\beta_{i}(\tau)[19]$.

Tuning the Vibration ${ }^{-}$-Feedback Controller. The asymptotic approximation of the system gives us a time invariant, averaged representation which can be used for controller tuning. However, even for such a small system, the controller tuning can be very difficult. The second order periodic controller has six adjustable parameters. A minimum requirement is that the closed loop must be stable, thus all closed loop poles must be placed in the LHP. Next, the poles of the controller should be placed in the LHP to maintain internal stability. It is also desirable to place the zeros in the LHP, otherwise there is not much point in using the periodic controller. Further restrictions on the location of the closed loop poles can be imposed to obtain better closed loop performance. In addition to the above guidelines it is essential to consider the physical constraints of the manipulated variable, such as saturation and unrealizable periods of oscillation in the case when there are hardware imposed limitations. The suggested method of tuning is to choose $\alpha$ and $F$ so that the controller poles are placed in the LHP. The system zeros may then be placed arbitrarily by selecting the coefficients of the numerator polynomial of the averaged open loop transfer function, since the coefficients are linear in the remaining controller parameters $h_{1}, h_{2}$, and $h_{1}^{1}$. To facilitate the closed loop pole placement, the original controller as proposed by Lee et al. [19] was modified to include a proportional gain term. This was done so that once the systems open loop poles and zeros were fixed, a single adjustable parameter was available to achieve more flexible control of the closed loop pole locations. Suitable choices of the controller poles and system zeros allow the system to be stable for all positive $\mathrm{k}$ with suitably small $\epsilon$ just as infinite gain margin can be achieved under the 
same conditions since only the combined gain of the controller/plant is of concern. Root locus techniques can then be used as a valuable tool in the design of periodic feedback controllers. It is clear that certain pole/zero configurations will lead to controllers that are stable for all positive $\mathrm{k}$, provided the original plant is stable, as is the case in this study. For example, an illustration of these ideas is shown in Figure 13a where the method was applied to design a periodic controller for a tubular reactor model. Placement of one zero to coincide with one plant pole, placement of the other zero to the left of the remaining plant pole and controller pole placement left of them results in an infinite gain margin for suitably small $\epsilon$.

\section{Vibrational-Feedback Control of a Tubular Reactor}

The effects of periodic feedback control on the tubular $\mathrm{CO}$ oxidation reactor model are investigated in order to assess gain margin improvements and transient behavior of the reactor.

The Reactor Model. The plant under consideration is the packed-bed tubular autothermal $\mathrm{CO}$ cxidation reactor modeled by 7 PDEs. The PDEs were discretized using orthogonal collocation, resulting in a large set of nonlinear ODEs in time. This model was then linearized about the operating condition of interest, resulting in a large set of linear ODEs. While this model is suitable for linear control system development, the order of the resulting periodic controller would be excessive, in fact, equal to the order of the plant, so model reduction is necessary. Reduced-order models of tise $\mathrm{CO}$ oxidation reactor were developed using the Generalized Structural Dominance Analysis (GSDA) $[20,21]$. The results indicate that a second order model with one RHP zero should do an adequate job of modeling the dynamic behavior of the tubular reactor model. The manipulated variable, quench flow, serves to decrease the inlet temperature to the reaction section and is a proven effective method for the control of such reactors [20]. Unfortunately, in some cases, this drop in inlet temperature results in a transient temperature rise in the downstream 

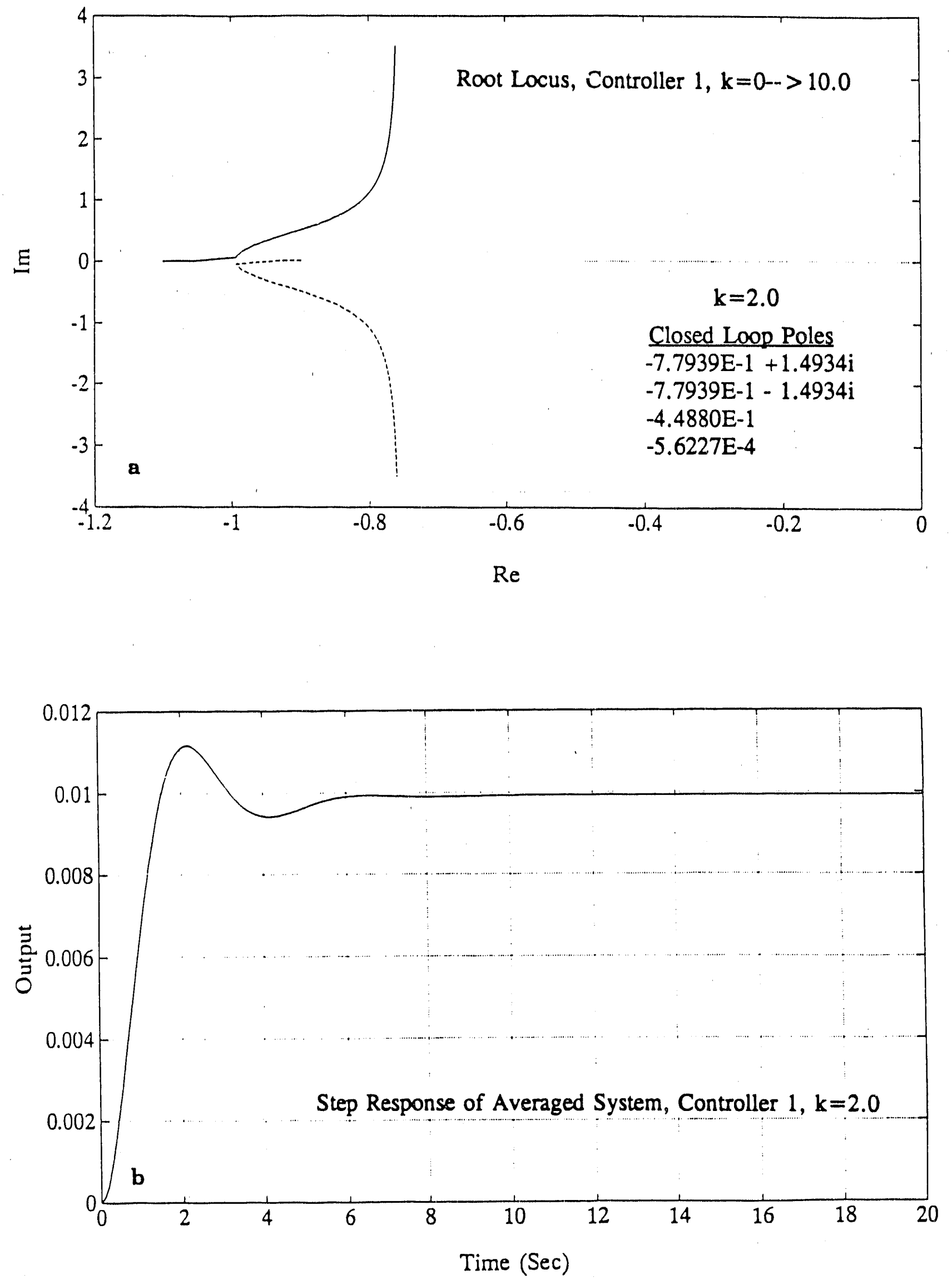

Figure 13. (a) Root locus diagram and (b) Step response of the averaged system for Controller 1 . 
section of the reactor. The mechanism that leads to this inverse response is the relative speed of propagation of the temperature and concentration gradients through the bed of the reactor. The resulting model thus has one finite RHP zero.

Dynamic Response of the Reactor. Use of the proposed tuning scheme resulted in several feasible sets of controller parameters. In the development of the controller for the reactor, one goal was to eliminate the inverse response and another was to increase the speed of the dynamic response to setpoint changes. To achieve these goals, the system zeros were placed in the left half plane and in fact, one was placed such that it canceled the plant pole closest to the imaginary axis, thus eliminating the slowest dynamics of the system. The set of controller parameters $\left(\alpha=-1.0, f_{1}=-0.618, f_{2}=-2.0, h_{1}=-27.78, h_{2}=\right.$ $\left.-1.0102 E+5, h_{1}^{1}=-1.1563 E+5\right)$, referred to as controller 1 , achieve these goals. The root locus and the averaged closed loop pole locations determined for a gain value of $k=2.0$ are given in Figure 13a. The step response of the averaged system is shown in Figure 13b, where output denotes exit concentration deviation. In all cases that follow, the magnitude of the step change is 0.01 , corresponding to a one percent change in the mole fraction of carbon monoxide. In the averaged system response, the inverse response is eliminated and the response is very fast. The simulation of the actual response of the system to a step change (Fig. 14) is highly oscillatory and overshoots 1000 percent. The settling time is very fast however and the system reaches the quasi-steady state in 10 seconds. Figure $14 \mathrm{~b}$ shows that an unrealistic amount of control action would be necessary to drive the system to setpoint this fast. Finally, from Figure 14c we note that the initial response of the system is in the negative direction, indicating that inverse response is still present in the periodic system. Clearly, controller 1 is far too demanding, so a more conservative design is in order.

Several other sets of controller parameters have been considered in Appendix 5 [3]. Extensive numerical studies indicated that larger values of $h_{1}$ and $\alpha$ improved the dynamic response by reducing the magnitude of the escillations in the output. The next design con- 

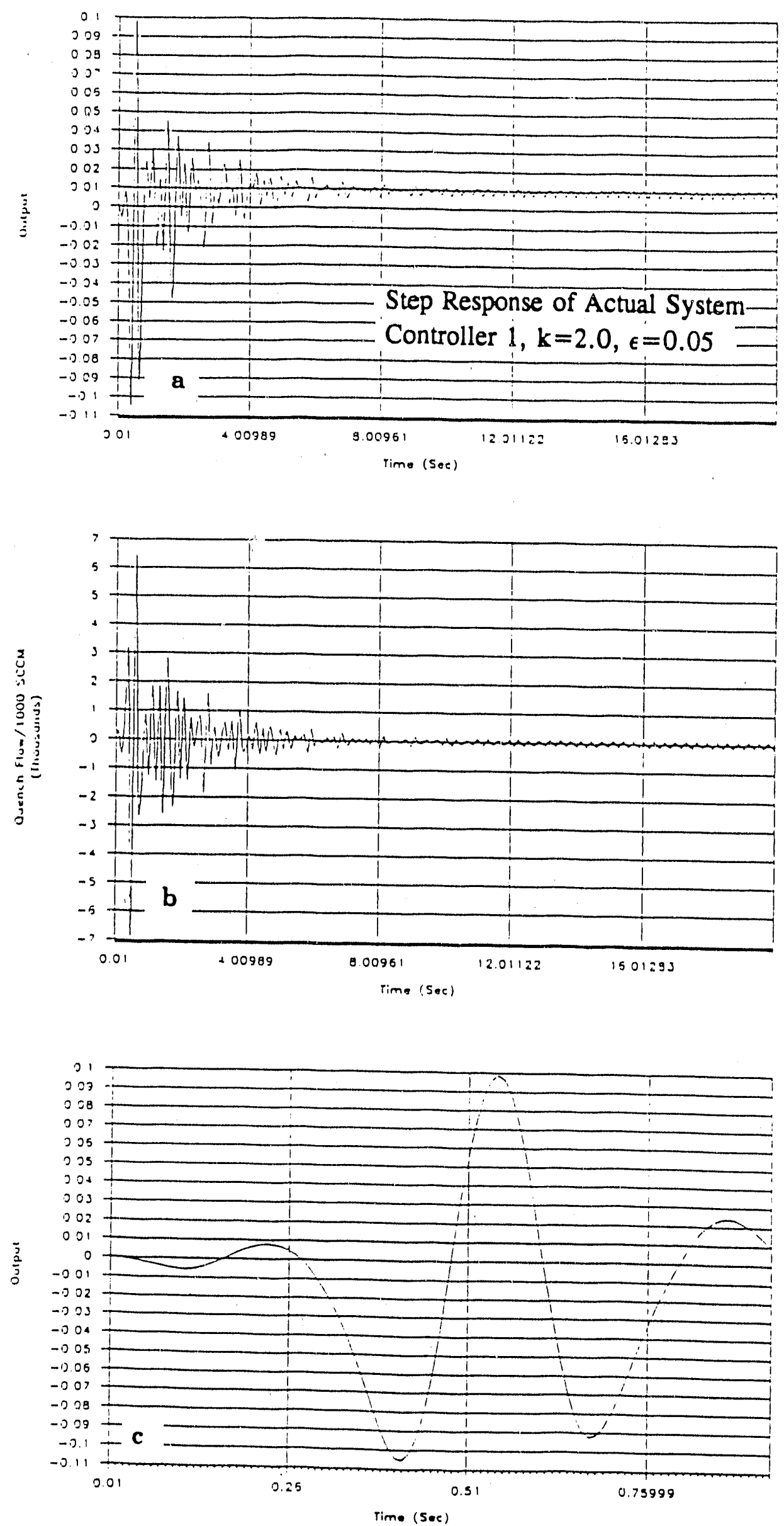

Figure 14. Response of the actual system to a step change: (a) Output, (b) quench flow (manipulated variable), (c) Output with an expanded time scale, Controller 1. 
sidered, controller 2 , utilized the set $\left(\alpha=2.0, f_{1}=-0.131, f_{2}=-1.0, h_{1}=-0.2, h_{2}=\right.$ $-19.1, h_{1}^{1}=-13.7$ ). The root locus (Fig. 15a) indicates the system is stable for all $\mathrm{k}$ and the averaged response for $\mathrm{k}=11.385$ is shown in Figure $15 \mathrm{~b}$. While the simulation results (Fig. 16) show that this is the most reasonable response observed, significant undershoot is still observed and the demand on the manipulated variable remains excessive.

Conclusions. It is clear that advantageous placement of the system zeros using vibrational-feedback can have a very positive impact in certain robustness qualities, such as the gain margin. Although our study focused on dynamic behavior, the gain margin property has been demonstrated in that for very large variations in the controller gain, the system response remains stable. For systems that are completely controllable and observable, an infinite gain margin can be guaranteed for suitably small values of $\epsilon$.

For the cases studied, periodic feedback seems to offer little advantages in improving the dynamic behavior of stable systems with right half plane zeros. Although all of the finite zeros of the system have been placed in the left half plane, it must be pointed out that this is an asymptotic property of the system. Figure 13c is a typical result of all controllers studied and shows that the system always exhibits inverse response in the early portion of the transient. In particular, the initial step that the system makes is of little concern if the oscillations of the controller drive the system in the opposite direction anyway.

For the cases studied, the demand on the manipulated variable's magnitude and period of oscillation were excessive. The required amplitude of oscillation is two to three orders of magnitude larger than the existing experimental system can handle. Also, the period of oscillation of the manipulated variable, in this case the quench flow rate, is a function of $\epsilon$. For typical values of $\epsilon$, the period is less than one second. Most control valves have time constants on the order of several seconds so for these reasons, experimental evaluation of these results is impossible at this time.

It has been demonstrated that the dynamic response of the periodic feedback system can be described reasonably well by the properties of the averaged system representation. 

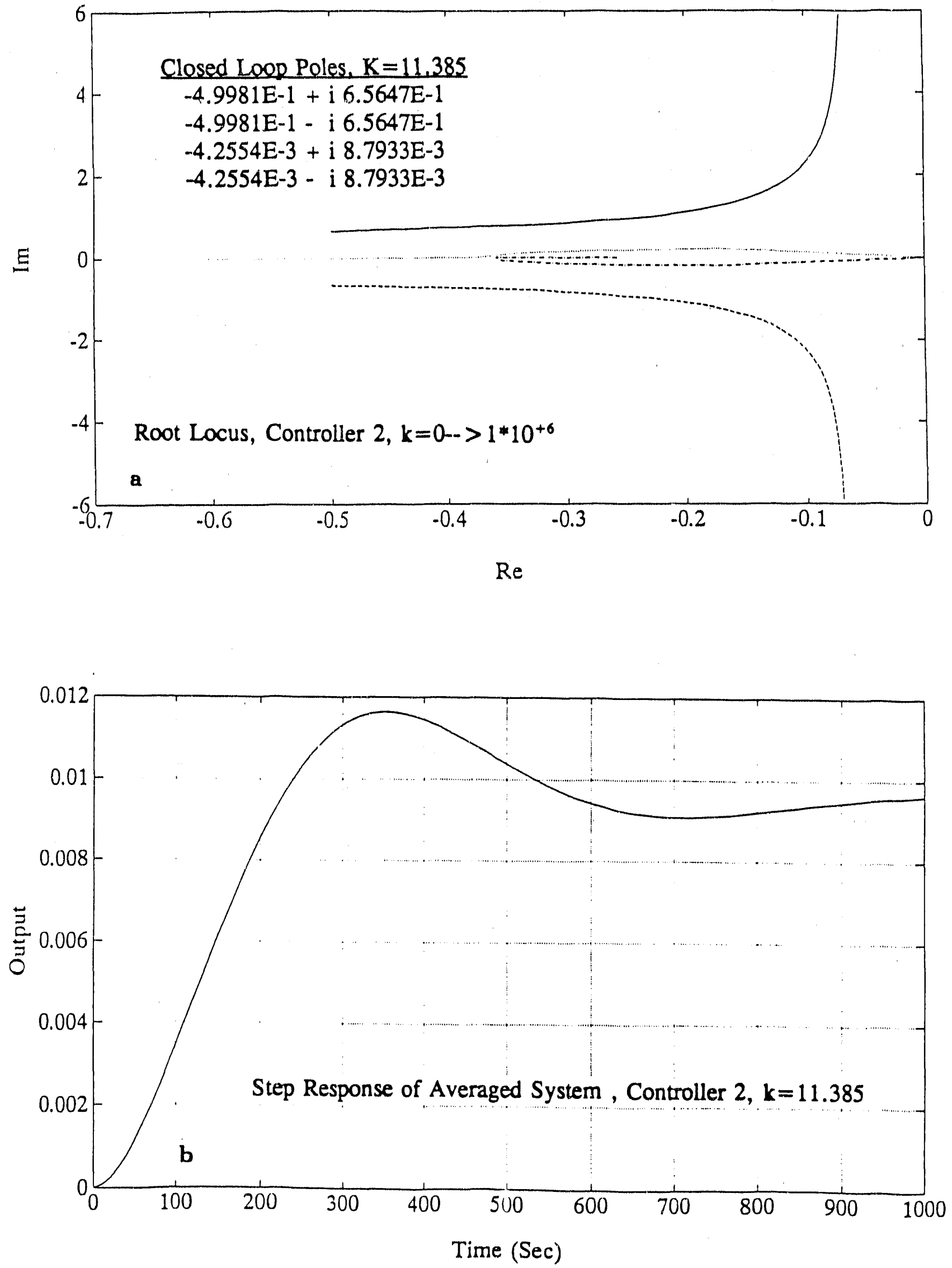

Figure 15. (a) Root locus diagram and (b) Step response of the averaged system for Controiler 2 . 

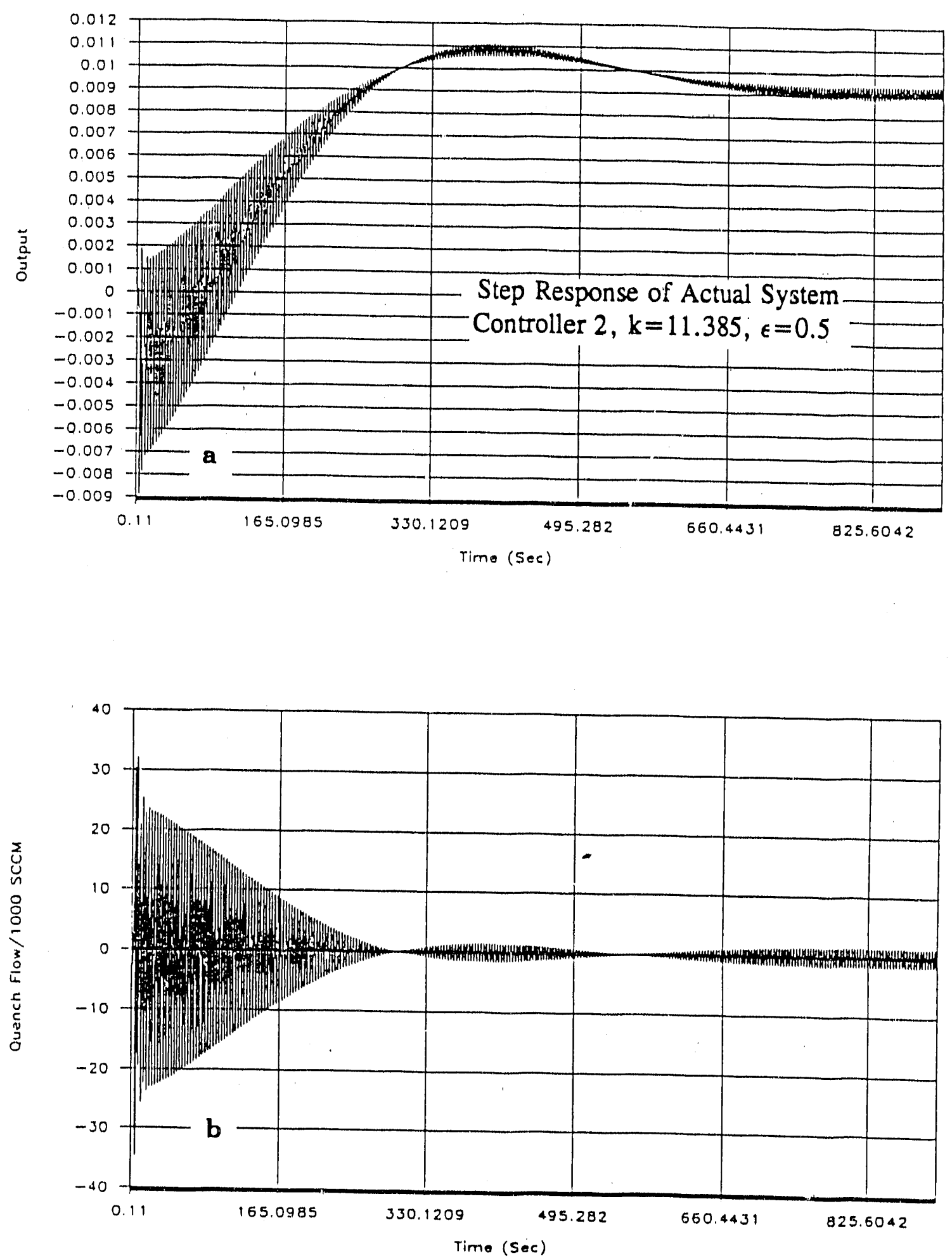

Figure 16. Response of the actual system to a step change: (a) Output, (b) quench flow (manipulated variable), Controller 2. 
In particular, if the magnitude of oscillation is not excessive, the properties of average offset, rise time, settling time and percent overshoot can be predicted without resort to costly numerical simulation of the periodic feedback system. It also has been shown that the root locus is a valuable tool in determining advantageous locations for zeros placement.

\section{CONCLUSIONS AND FUTURE RESEARCH DIRECTIONS}

\section{Conclusions}

The studies conducted during this project have shown that forced periodic operation of chemical reactors cause improvements in conversion, selectivity and yield. Since the periodic forcing policies are implemented by periodic adjustment of the valve openings that regulate reactant flow rates, their impact on operating costs are minimal. Consequently, the increase in yield improves both the productivity and the profitability. In a test case where ethylene is converted to ethylene oxide by partial oxidation, 23 percent yield improvement was attained with respect to steady state operation at the same conditions, by simultaneous periodic forcing of feed flow rate and ethylene feed composition.

A number of issues must be resolved while searching for a forced periodic operation policy that will enhance productivity. First, the optimal steady state operating policy must be found and the existence and proximity of other attracting points in the vicinity of the steady state must be studied. Next, the existence of stable limit cycles around the optimal steady state operating point must be studied. Then the optimal periodic forcing parameters that maximize the reactor yield must be searched. Focusing on a search domain in the vicinity of the optimal steady state rather than conducting a global optimization study is dictated by the size of the optimization problem. Although the formulation of the global optimization problem is straightforward, the numerical burden of finding the stable limit cycles of systems represented by a large number of stiff differential equations and globally optimizing with respect to several periodic forcing parameters is too large.

Several novel techniques have been proposed and various computer programs have 
been developed during this project. All program development activities were channeled towards the generation of computer codes which were independent of specific reactions and reactors. This permits the use of singularity and bifurcation analysis tools, and periodic forcing policy optimization techniques with any reaction and any reactor configuration. As a result of this philosophy, the numerical tools were preferred over the analysis tools based on the method of averaging which necessitate system specific derivations. The techniques developed provide the building blocks to address the issues listed in the previous paragraph. The numerical singularity and bifurcation analy sis techniques discussed in Chapter III can classify all stedy state behaviors that can be exhibited by a specific reactor and find all attractors corresponding to a specific set of operating conditions. They have been used successfully for lumped parameter (CSTR) and distributed parameter (tubular) reactor systems. The programs based on the numerical method for determining optimal parameter values in forced periodic operation (Chapter II) can search for the stable limit cycles due to periodic forcing and carry out multivariable constrained optimization to find optimal forcing parameters. They have been tested with lumped parameter systems with multiple reactions and show good promise for extending the approach to distributed parameter systems.

Vibrational-feedback control approach was used to improve the transient reactor behavior by eliminating right half-plane (RHP) zeros. An odd number of RHP zeros cause inverse response behavior and provide a challenging control problem. Vibrational-feedback improved robustness by increasing the gain margin and eliminated the inverse response of the averaged system. However, the actual system continued to preserve the inverse response and the demand on the manipulated variable actions were too excessive.

The impact of forced periodic operation policies on improving stationary state properties such as average yield outweight their effect to dynamic behavior. This fiis well with many chemical process activities where cortinuous production at a specific set of operating conditions is desired and regulatory control is utilized for disturbance rejection. 
Consequently, a forced periodic operation policy which does not drive the system towards an undesirable attractor and which improves yield over the steady state operation can be implemented.

\section{Future Research Directions}

This project has generated several numerical tools for analyzing steady state and periodically forced behavior of chemical systems and has illustrated the potential benefits of forced periodic operation. It has also shown the need for a thorough analysis before embarking on a periodic operation policy, in order to discover the existence of dangerous attractors and to prevent reduction of yield by selecting poor periodic forcing policies. The future research tasks are to extend and perfect these tools to carry out a complete analysis of chemical reactor systems commonly found in industry. This involves innovative extension of the numerical singularity and bifurcation tools, development of the optimization tools for selecting optimal periodic forced operation parameters for distributed parameter systems, and compilation of case studies with different generic features commonly found in large throughput industrial reactors. Considering the computational burden associated with such studies, use of parallel processing techniques and supercomputer resources as appropriate is necessary. Consequently, computer code generation which utilizes such tools should also be part of the future activities.

Use of the numerical bifurcation and singularity analysis tools to assess the behavior of reactors subject to forced periodic operation will provide the ranges of operating conditions leading to qualitatively different behavior under periodic forcing. Furthermore, local studies at critical locations such as turning points and inflection points (Figure 11) can indicate semi-qualitatively how periodic forcing can affect the bifurcation behavior. The goal must be the development of techniques that can provide complete and reliable information as an alternative to time consuming dynamic simulation studies that yield only partial information. 
Techniques for selecting optimal periodic forced operation parameters of tubular reactors must be developed. In this regard, the extension of the shooting algorithm to distributed parameter systems shows good promise.

Innovative use of coarse parallel processing techniques has resulted in severalfold speed increases in numerical singularity analysis and bifurcation analysis routines. The same approach should be used in singularity analysis of periodically forced systems. Similar paths to parallelization must be sought in the optimization tools. Vectorization and use of supercomputers would also provide speed increases needed for addressing optimization of forced periodic operation of tubular packed-bed reactor systems. In future studies both parallel processing and vectorization must be considered.

Case studies must be conducted for testing the applicability and robustness of the techniques and computer codes developed. Different types of reaction systems encountered in the industry should be studied. For example, ethylene oxidation and phthallic anhydride production reactors would represent partial oxidation reactions with several competing steps carried out in multi-bed tubular reactors, while ammonia synthesis would represent equilibrium limited reactions in large packed beds connected in series.

The ultimate goals must be to reduce guesswork in technical decision making and to eliminate unnecessary conservativeness in operating policies. This study has generated and demonstrated tools which provide a clear global picture of reactor behavior for assessment of the effects of operating conditions and selection of safe and optimal operating policies. Continued work in this area would extend the power of these tools to meet the challenges of more complex reactor systems of industrial importance.

\section{LIST OF PUBLICATIONS AND PRESENTATIONS RESULTING FROM THE PROJECT AND EDUCATIONAL ACTIVITIES}

\section{Publications and Manuscripts}

Chylla R. W. and A. Çinar: Synthesis of Model-Based Controllers for an Autothermal Reactor, Can. J. Chem. Eng, 68,666, 1990.

Chylla R. W. and A. Çinar: Design of Resilient Controllable Chemical Processes: An Autothermal Reactor Case Study, Ind. Eng. Chem. Res., 28, 1218, 1990. 
Adomaitis R. A. and A. Cinar: Numerical Singularity Analysis. Chem. Eng. Sci, Accepted for publication, 1990. (Appendix 3).

Özgülşen F., K. Rigopoulos and A. Cinar: A Comparative Study of Tools for Assessing the

Effects of Forced Periodic Operation of Catalytic Reactors, submitted, 1990. (Appendix 1).

Kendra S. and A. Çinar: Dynamic Behavior of Periodic Feedback Control Systems, submitted to Chem. Eng. Commun., 1990. (A ppendix 5).

Özgülşen F. and A. Çinar: A Numerical Method for Determining Optimal Forcing Parameters, in preparation (1990). (Appendix 2).

\section{Conference Preprints and Proceedings}

Adomaitis R. A. and A. Çinar: The Bifurcation Behavior of an Autothermal Packed-Bed Reactor, Proc. 1987 Ameri. Cntrl Conf., 1419, 1987.

Chylla R. W. and A. Çinar: Experimental Control of an Autothermal Packed-bed Reactor, Proc. 1987 Ameri. Cntrl Conf., 313, 1987.

Adomaitis R. A. and A. Chinar: The Bifurcation Behavior of an Autothermal Tubular Reactor. Proc. IFAC '87 10th World Congress on Autom. Cntrl, 2, 212, 1987.

Adomaitis R. A. and A. Çinar: Numerical Singularity Analysis of Feedback Controlled Chemical Reactors. Proc. 1988 Amer. Control Conf., 1779, 1988. (Appendix 5).

Adomaitis R. A. and A. Çinar: Numerical Bifurcation Analysis of Large Nonlinear Systems On A Multiprocessor, in Parallel Processing for Scientif c Computing, SIAM Press, in print, 1990.

\section{Lectures and Presentations}

Chylla R.W. and A. Çinar: Experimental Control of an Autothermal Packed Bed Reactor, 1987 Ameri. Control Conf., Minneapolis, MN.

Chylla R. W. and A. Çinar: Experimental Control of an Autothermal Packed-bed Reactor, 1987 Ameri. Control Conf., Minneapolis, MN.

Adomaitis R.A. and A. Çinar: The Bifurcation Behavior of an Autothermal Tubular Reactor, IFAC '87 10th World Congress on Autom. Cntrl.,Munich, FRG

Rigopoulos K., X. Shu and A. Çinar: Vibrational Control Approach for Stabilization and Productivity Improvement in Chemical Reactors, Paper 20c, 1987 AIChE Annual Meeting, New York, NY, 1987

Adomaitis R. A. and A. Çinar: The Bifurcation of Feedback-Controlled Chemical Reactors, Paper 84c, 1987 AIChE Annual Meeting, New York, NY, 1987.

Adomaitis R. A. and A. Cinar: Numerical Singularity Analysis of Feedback Controlled Chemical Reactors, 1988 Ameri. Control Conf., Atlanta, GA, 1988.

Adomaitis R. A. and A. Çinar: Numerical Bifurcation Analysis and Parallel Processing, Paper 174g, 1988 AIChE Annual Meeting, Washington, D.C., 1988.

Kendra S. and A. Çinar: Periodic Output Controllers for Tubular Packed-Bed Reactors, SIAM Conf. on Control in the 90's, San Francisco, CA, 1989.

Adomaitis R. A. and A. Cinar: Numerical Bifurcation Analysis of Large Nonlinear Systems On A Multiprocessor, SIAM Fourth Conf. on Parallel Processing for Scientific Computing Conf., Chicago, IL, 1989. 
Rigopoulos K., F. Özgülşen, A. Çinar and R. Adomaitis: Numerical Tools for Assessing the Effects of Forced Periodic Chemical Reactor Operation, Paper 128g, 1989 AIChE Annual Meeting, San Francisco, CA, 1989.

Özgülşen F., R. A. Adomaitis and A. Çinar: A Numerical Method for Determining Optimal Forcing Parameters, 1990 AIChE Annual Meeting, Paper 311-11, Chicago, IL, 1990.

\section{Educational Activities}

This project contributed to five $\mathrm{Ph} . \mathrm{D}$. projects:

Chylla, R. W., Design and control of tubular autothermal reactors (Ph.D. May 87)

Adomaitis, R. A., The bifurcation and modification of equilibrium states in chemical reactors (Ph.D. May 88).

Rigopoulos K., Selection and Yield Improvement by Forced Periodic Operation: Ethylene Oxidation Reaction (Ph.D. May 1990).

Kendra S., Advanced Control System Development for Multivariable Chemical Processes (Ph.D. in progress).

Özgülşen F., Optimization of Chemical Reactor Operation by Forced Periodic Policies (Ph.D. in progress). 


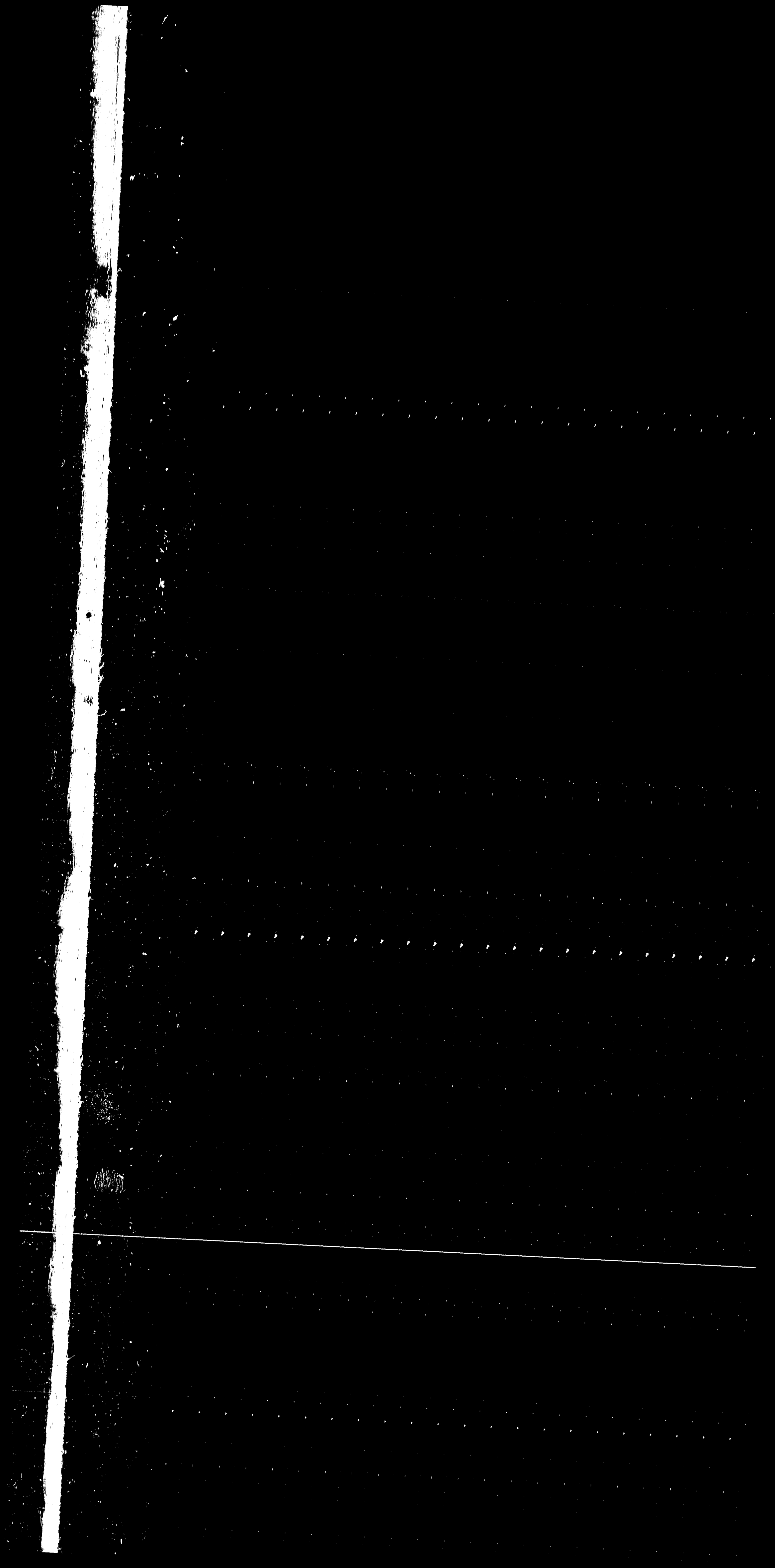




\section{REFERENCES}

[1] Adomaitis R. A. and A. Cinar: Numerical Singularity Analysis. Chem. Eng. Sci., Accepted for publication, 1990. (Appendix 3).

[2] Ozgulsen F., K. Rigopoulos and A. Çinar: A Comparative Study of Tools for Assessing the Effects of Forced Periodic Operation of Catalytic Reactors, submitted, 1990. (Appendix 1).

[3] Kendra S. and A. Çinar: Dynamic Behavior of Periodic Feedback Control Systems, submitted to Chem. Eng. Commun., 1990. (Appendix 5).

[4] Bogoliubov N. N. and Yu. A. Mitropolsky: Asymptotic Methods in the Theory of Nonlinear Oscillations, Gordon and Breach, New York, 1961.

55] R. Bellman, J. Bentsman and S. M. Meerkov: Vibrational control of nonlinear systems: Vibrational stabilizability, IEEE Trans. Automat. Contr., AC-31, 710, 1986; Vibrational control of nonlinear systems: Vibrational controllability and transient behavior, IEEE Trans. Automat. Contr., AC-31, 717, 1986.

[6] DOE Grant No: DE-FG02-84ER13205

(7) A. Çinar, J. Deng, S. M. Meerkov and X. Shu: Vibrational control of an exothermic reaction in a CSTR: Theory and experiments, AIChE J., /bf 33, 353, 1987.

[8] A. Cinar, K. Rigopoulos, S. M. Meerkov and X. Shu: Vibrational control of chemical reactors: Stabilization and conversion improvement in an exothermic CSTR, Chem. Eng. Commun.., 59, 299, 1987.

(9) Rigopoulos K, X. Shu and A. Cinar: Forced Periodic Control of an Exothermic CSTR with Multiple Input Oscillations, AIChE J., 34, 2041, 1988.

[10] Carra S. and P. Forzatti: Engineering Aspects of Selective Hydrocarbon Oxidation, Cat. Rev. - Sci. Eng., 15, 1, 1977.

(11] Leis, J. R. and M. A. Kramer: The Simultaneous Solution and Sensitivity Analysis of Systems Described by Ordinary Differential Equations, ACM Trans. on Math. Software, 14, 45, 1988.

[12] Ozgulsen F. and A. Çinar: A Numerical Method for Determining Optimal Forcing Parameters, in preparation (1990). (Appendix 2).

[13] Golubitsky, M. and D. G. Schaeffer, Singularities and Groups in Bifurcation Theory, Volıme I. Springer-Verlag, New York, 1985.

[14] Adomaitis R. A. and A. Cinar: The Bifurcation Behavior of an Autothermal PackedBed Tubular Reactor, Chem. Eng. Sci., 43 887, 1988.

[15] Adomaitis, R. A., The bifurcation and modification of equilibrium states in chemical reactors, $\mathrm{Ph} . \mathrm{D}$. Thesis, Illinois Institute of Technology, 1988.

[16] Keller H. B., Numerical solution of bifurcation and eigenvalue problems. In Applications of Bifurcation Theory (Edited by Rabinowitz P. H.). Academic Press, New York, 359-384, 1977.

[17] Adomaitis R. A. and A. Çinar: Numerical Bifurcation Analysis of Large Nonlinear Systems On A Multiprocessor, SIAM Fourth Conf. on Parallel Processing for Scientific Computing Conf., Chicago, IL, 1989.

[18] Adomaitis R. A. and A. Cinar: Numerical Singularity Analysis of Feediback Controiled Chemical Reactors. Proc. 1988 Amer. Control Conf., 1779, 1988 (Appendix 5). 
[19] Lee S., S. M. Meerkov and T. Runolfsson: Vibrational-Feedback Controllers: Zero Placement Capabilities, IEEE Trans. Autom. Cntrl, AC-32, 604, 1987.

[20] Chylla R. W. and A. Çinar: Synthesis of Model-Based Controllers for an Autothermal Reactor, Can. J. Chem. Eng, 68, 666, 1990.

[21] Chylla R. W. and A. Çinar: Design of Resilient Controllable Chemical Processes: An Autothermal Reactor Case Study, Ind. Eng. Chem. Res., 28, 1218, 1990. 


\begin{abstract}
The effects of vibrational control on selectivity and yield of complex chemical reactions and on tubular packed-bed reactor behavior have been studied.

i. Selectivity and yield improvement by forced periodic operation of complex chemical systems have been assessed by vibrational control approach and by a numerical approach. Vibrational control approach has provided good insight. The numerical approach is based on the computation of stable limit cycles by using a shooting algorithm. Since the reactor model is incorporated as a subroutine to the computer code developed, effects of forced periodic operation on various types of reactions and reactors can be assessed with ease. The program has been integrated in an optimization package for the selection of optimal operating conditions for maximizing the reactor yield.

The forced periodic operation of ethylene oxidation reaction system, a hydrocarbon partial oxidation reaction of commercial importance, has been studied using both techniques. Yield improvement of 23 percent has been attained by periodic forcing of feed flow rate and ethylene feed concentration.

ii. A numerical singularity analysis technique has been developed and applied to openloop and feedback controlled tubular packed-bed reactors. The technique is applicable to various kinds of reactor systems and it can be used to classify their qualitatively different static bifurcation behaviors as a function of input variables such as feed temperature and concentration. Such a classification displays the existence of other attractors in the vicinity of the steady state around which a reactor would be subjected to forced periodic operation. Preliminary studies have been conducted to extend the technique to analyze and classify the bifurcation behavior of chemical reactors under vibrational control.

iii. Low order vibrational-feedback controllers have been designed and a new tuning method has been used in vibrational-feedback control of chemical reactors. Vibrationalfeedback controllers allow zero relocation and consequently have the potential of eliminating inverse response behavior due to right half-plane zeros. A vibrational-feedback controller based on a reduced-order model of the $\mathrm{CO}$ oxidation reactor has greatly improved the gain margin and improved the averaged dynamic behavior. However, the inverse response behavior persisted in dynamic simulations of the actual system and excessive moves were expected of the manipulated variable.
\end{abstract}



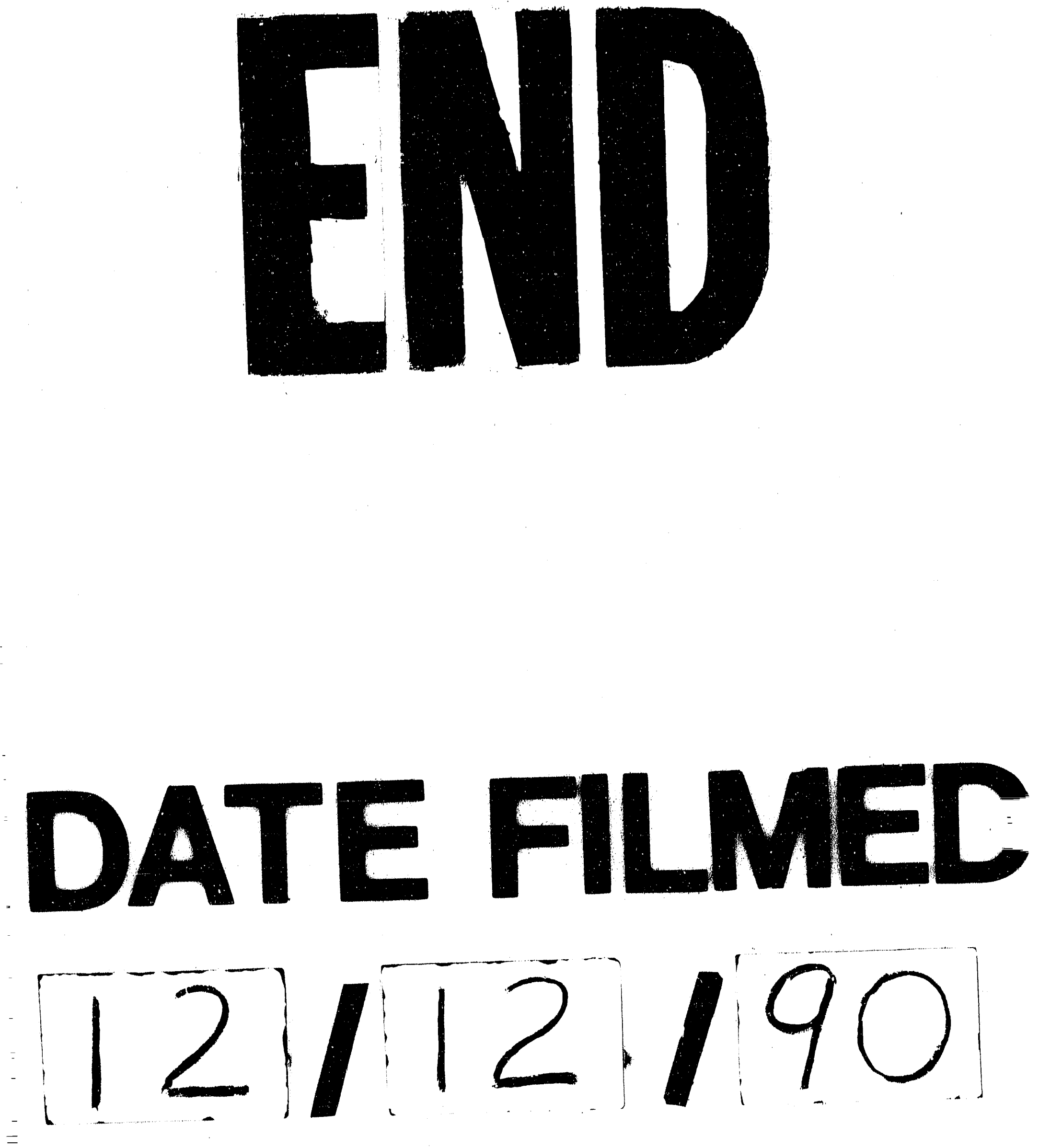
